TRANSACTIONS OF THE

AMERICAN MATHEMATICAL SOCIETY

Volume 360, Number 7, July 2008, Pages 3365-3389

S 0002-9947(08)04339-0

Article electronically published on February 27, 2008

\title{
BRAIDS, TRANSVERSAL LINKS AND THE KHOVANOV-ROZANSKY THEORY
}

\author{
HAO WU
}

\begin{abstract}
We establish some inequalities for the Khovanov-Rozansky cohomologies of braids. These give new upper bounds of the self-linking numbers of transversal links in standard contact $S^{3}$ which are sharper than the well-known bound given by the HOMFLY polynomial. We also introduce a sequence of transversal link invariants and discuss some of their properties.
\end{abstract}

\section{INTRODUCTION}

In [7, 8], M. Khovanov and L. Rozansky introduced two versions of KhovanovRozansky link cohomologies. Their construction is based on matrix factorizations associated to certain planar diagrams. The version in [7] uses the potential $x^{n+1}$, which leads to a $\mathbb{Z} \oplus \mathbb{Z}$-graded cohomology theory $H_{n}$ for each $n \geq 2$. The version in 8] uses the potential $a x$, which gives a $\mathbb{Z} \oplus \mathbb{Z} \oplus \mathbb{Z}$-graded cohomology theory $H$.

M. Khovanov and L. Rozansky showed that $H_{n}$ is invariant under Reidemeister moves, and the isomorphism type of $H$ is invariant under Markov moves of closed braids up to overall shifts of gradings. The graded Euler characteristics of $H_{n}$ and $H$ are variants of the HOMFLY polynomial.

In this paper, we discuss some applications of these theories in contact topology, specifically the transversal knot theory. The sign convention of this paper is given in Figure 1, which is opposite to that used in [7, 8.
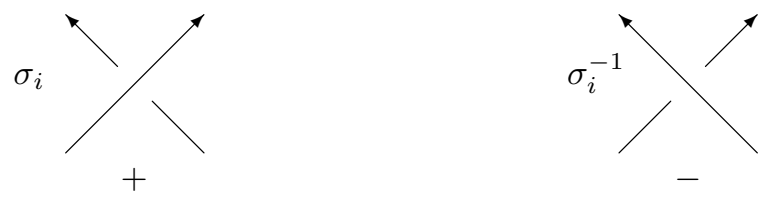

FiguRE 1. Sign of a crossing

First, we introduce a variant of $H$ whose graded isomorphism type is invariant under Reidemeister moves. Let $B$ be a closed braid with $b$ strands and writhe $w$. Define $\hat{H}(B)$ to be $H(B)$ with each of the two quantum gradings shifted by $\frac{w+b}{2}$ and the homological grading shifted by $-\frac{w+b}{2}$. Then $\hat{H}(B)$ is $\frac{1}{2} \mathbb{Z} \oplus \frac{1}{2} \mathbb{Z} \oplus \frac{1}{2} \mathbb{Z}$-graded. From M. Khovanov and L. Rozansky's construction, it's clear that the gradings of $\hat{H}(B)$ are now invariant under all Markov moves.

Received by the editors January 20, 2006 and, in revised form, May 24, 2006.

2000 Mathematics Subject Classification. Primary 57M25, 57R17.

Key words and phrases. Braid, transversal knot, knot homology, matrix factorization.

(C)2008 American Mathematical Society Reverts to public domain 28 years from publication 
Denote by $H_{n}^{j, k}$ the space of homogeneous elements with grading $(j, k)$ of $H_{n}$, where $j$ is the homological grading, and $k$ is the quantum grading which comes from the exponents of the markings $x_{i}$. Denote by $\hat{H}^{j, k, l}(B)$ the space of homogeneous elements with grading $(j, k, l)$ of $\hat{H}(B)$, where $j$ is the cohomological grading, $k$ is the quantum $a$-grading which comes from the exponents of the formal variable $a$, and $l$ is the quantum $x$-grading which comes from the exponents of markings $x_{i}$.

For a link $L$, define

$$
\begin{gathered}
g_{\text {max }}^{(n)}(L)=\max \left\{k \mid H_{n}^{j, k}(L) \neq 0 \text { for some } j\right\}, \\
g_{\min }^{(n)}(L)=\min \left\{k \mid H_{n}^{j, k}(L) \neq 0 \text { for some } j\right\}, \\
\hat{g}_{\max }(L)=\max \left\{k \mid \hat{H}^{j, k, l}(L) \neq 0 \text { for some } j, l\right\}, \\
\hat{g}_{\min }(L)=\min \left\{k \mid \hat{H}^{j, k, l}(L) \neq 0 \text { for some } j, l\right\} .
\end{gathered}
$$

These are numerical link invariants. We have:

Theorem 1.1. If $B$ is a closed braid with $b$ strands and writhe $w$, then

$$
\frac{w-b}{2} \leq \hat{g}_{\min }(B) \leq \hat{g}_{\max }(B) \leq \frac{w+b}{2}-1 \text {. }
$$

If we further assume that the numbers of positive and negative crossings of $B$ are $c_{+}$and $c_{-}$, respectively, then

$$
(n-1)(w-b)-2 c_{-} \leq g_{\min }^{(n)}(B) \leq g_{\max }^{(n)}(B) \leq(n-1)(w+b)+2 c_{+} .
$$

Definition 1.2. The braid number $b(L)$ of a link $L$ is the minimal number of strands necessary to represent $L$ as a braid.

The next corollary follows easily from Theorem 1.1

Corollary 1.3. For a link L,

$$
\begin{gathered}
b(L) \geq \hat{g}_{\max }(L)-\hat{g}_{\min }(L)+1, \\
b(L) \geq \frac{1}{2} \limsup _{n \rightarrow \infty} \frac{g_{\max }^{(n)}(L)-g_{\min }^{(n)}(L)}{n-1} .
\end{gathered}
$$

Theorem 1.4. If $L$ is a transversal link in the standard contact 3-sphere, then

$$
\begin{gathered}
s l(L) \leq 2 \hat{g}_{\min }(L), \\
s l(L) \leq-\limsup _{n \rightarrow \infty} \frac{g_{\max }^{(n)}(\bar{L})}{n-1},
\end{gathered}
$$

where "sl" is the self-linking number, and $\bar{L}$ is the mirror image of $L$.

Therefore, if $K$ is a Legendrian knot in the standard contact 3-sphere, then

$$
\begin{gathered}
t b(K)+|r(K)| \leq 2 \hat{g}_{\min }(K), \\
t b(K)+|r(K)| \leq-\limsup _{n \rightarrow \infty} \frac{g_{\max }^{(n)}(\bar{K})}{n-1},
\end{gathered}
$$

where " $t b$ " is the Thurston-Bennequin number, and " $r$ " is the rotation number.

Proof. By [2], any transversal knot is transversally isotopic to a transversal braid, and the self-linking number $\operatorname{sl}(B)$ of a transversal braid $B$ with $b$ strands is equal to $w-b$, where $w$ is the writhe of $B$. So Theorem 1.1 implies the first part of the theorem. The second part follows from the first part by a push-off argument given in $[2]$. 
In [1], O. Plamenevskaya constructed an invariant $\psi$ of transversal links in standard contact $S^{3}$ using the original Khovanov homology $\mathcal{H}$ defined in [6]. We generalize her construction, and, for each $n \geq 2$, define an invariant $\psi_{n}$ of transversal links in standard contact $S^{3}$ using $H_{n}$.

Theorem 1.5. Let $L$ be a transversal link in the standard contact $S^{3}$. For each $n \geq 2$, we can associate to $L$ an element $\psi_{n}(L)$ of $H_{n}(\bar{L})$ of cohomological degree 0 and quantum degree $-(n-1) \operatorname{sl}(L) . \psi_{n}(L)$ is invariant under transversal isotopy of $L$ up to multiplication by a non-zero scalar.

When $n=2, \psi_{2}(L)$ is identified with $\psi(L)$ under the isomorphism

$$
H_{2}(\bar{L}) \cong \mathcal{H}(L) \otimes_{\mathbb{Z}} \mathbb{Q}
$$

Comparing Theorems 1.4 and 1.5, it's easy to see that the sequence $\left\{\psi_{n}\right\}$ sometimes detects the maximal self-linking number. In particular, we can generalize Plamenevskaya's computation for quasi-positive braids and get a stronger conclusion.

Corollary 1.6. If $L$ is a transversal link in the standard contact $S^{3}$ with $\psi_{n}(L) \neq 0$ for infinitely many $n$, then

$$
S L(L)=\operatorname{sl}(L)=-\limsup _{n \rightarrow \infty} \frac{g_{\max }^{(n)}(\bar{L})}{n-1},
$$

where $S L(L)$ is the maximal self-linking number of all transversal links smoothly isotopic to $L$.

In particular, if a transversal link $L$ is transverse isotopic to a quasi-positive transversal braid, then $\psi_{n}(L) \neq 0, \forall n \geq 2$, and, thus, the above equation is true.

Proof. The first part of the corollary is clear from Theorems 1.4 and 1.5. The second part will be proved in Proposition 4.5.

A transversal knot invariant is said to be non-classical if it is not determined by the smooth knot type and the self-linking number. There are still no known examples of efficient non-classical transversal knot invariants.

Conjecture 1.7. The sequence $\left\{\psi_{n}\right\}$ is a non-classical transversal knot invariant.

The rest of this paper is organized as follows. In Section 2, we briefly review the definitions of the Khovanov-Rozansky cohomologies and compare the graded Euler characteristics of these cohomologies with the HOMFLY polynomial, which explains why Theorems 1.1, 1.4 and Corollary 1.3 imply the corresponding results by J. Franks, R. Williams and H. Morton in 4, 9, including the upper bound of the self-linking number from the HOMFLY polynomial. In Section 3, we study the Khovanov-Rozansky cohomologies of closed braids and prove Theorem 1.1. In Section 4, we construct the invariants $\psi_{n}$ and study some of their properties.

\section{Khovanov-Rozansky TheORY}

2.1. Matrix factorizations. Let $R=\mathbb{Q}\left[t_{1}, \cdots, t_{m}\right]$ be a polynomial ring. $\mathrm{A}$ matrix factorization $M$ over $R$ with potential $w \in R$ is a collection of two $R$-modules $M^{0}, M^{1}$ and two module homomorphisms $d^{0}: M^{0} \rightarrow M^{1}, d^{1}: M^{1} \rightarrow M^{0}$, called differential maps, s.t.,

$$
d^{1} d^{0}=w \cdot \operatorname{id}_{M^{0}}, \quad d^{0} d^{1}=w \cdot \operatorname{id}_{M^{1}} .
$$


We usually write such a matrix factorization $M$ as

$$
M^{0} \stackrel{d_{0}}{\longrightarrow} M^{1} \stackrel{d_{1}}{\longrightarrow} M^{0} .
$$

For $a, b \in R$, denote by $(a, b)_{R}$ the matrix factorization

$$
R \stackrel{a}{\rightarrow} R \stackrel{b}{\rightarrow} R
$$

where $a, b$ act on $R$ by multiplication. This matrix factorization has potential $a b$. When the polynomial ring $R$ is clear from the context, we drop it from the notation, and only write $(a, b)$.

Denote by

$$
\left(\begin{array}{cc}
a_{1} & b_{1} \\
a_{2} & b_{2} \\
\vdots & \vdots \\
a_{k} & b_{k}
\end{array}\right)_{R}
$$

the tensor product of $\left(a_{1}, b_{1}\right)_{R},\left(a_{2}, b_{2}\right)_{R}, \cdots,\left(a_{k}, b_{k}\right)_{R}$, where the differential maps are given by the (signed) Leibniz rule. Its potential is

$$
w=a_{1} b_{1}+a_{2} b_{2}+\cdots+a_{k} b_{k} .
$$

Again, when $R$ is clear from the context, we drop it from the notation.

2.2. Grading shifts. Suppose $M$ is a $\mathbb{Z}$-graded module over a $\mathbb{Z}$-graded ring, and $m \in \mathbb{Z}$. Define $M\{m\}$ to be $M$ with grading shifted by $m$, i.e., an element $x$ of $M\{m\}$ is homogeneous of degree $i+m$ if and only if it's a homogeneous element of $M$ of degree $i$. Similarly, suppose $M$ is a $\mathbb{Z} \oplus \mathbb{Z}$-graded module over a $\mathbb{Z} \oplus \mathbb{Z}$-graded ring, and $m, l \in \mathbb{Z}$. Define $M\{m, l\}$ to be $M$ with grading shifted by $(m, l)$, i.e., an element $x$ of $M\{m, l\}$ is homogeneous of bi-degree $(i+m, j+l)$ if and only if it's a homogeneous element of $M$ of degree $(i, j)$.

2.3. Planar diagrams. To construct the Khovanov-Rozansky cohomology theories, one considers the planar diagrams $\Gamma$ with the following properties:

(1) $\Gamma$ consists of two types of edges: regular edges and wide edges. These edges intersect only at their endpoints.

(2) Regular edges are disjoint from each other. Wide edges are disjoint from each other.

(3) Each regular edge is oriented, and contains at least one marked point. Open endpoints of regular edges are marked.

(4) Each wide edge has exactly two regular edges entering at one endpoint, and exactly two regular edges exiting from the other endpoint.

See Figures 7, 8, 9,10 below for examples of such diagrams.

2.4. Matrix factorization associated to a planar diagram. Let $\Gamma$ be a planar diagram satisfying the conditions in subsection 2.3. and let $\left\{x_{1}, \cdots, x_{p}\right\}$ be the set of markings on $\Gamma$. Let $R^{\prime}=\mathbb{Q}\left[x_{1}, \cdots, x_{p}\right]$ be the $\mathbb{Z}$-graded polynomial ring so that $\operatorname{deg} x_{i}=2, \forall i$. Let $R=\mathbb{Q}\left[a, x_{1}, \cdots, x_{p}\right]$ be the $\mathbb{Z} \oplus \mathbb{Z}$-graded polynomial ring so that $\operatorname{deg} a=(2,0)$ and $\operatorname{deg} x_{i}=(0,2), \forall i$.

For an oriented (regular) arc $L_{j}^{i}$ in $\Gamma$ from the point marked by $x_{j}$ to the point marked by $x_{i}$ (see Figure 2) that has no marked interior points, let $C_{n}\left(L_{j}^{i}\right)$ be the 


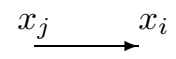

FIGURE 2. $L_{j}^{i}$

matrix factorization $\left(\pi_{i j}, x_{i}-x_{j}\right)_{R^{\prime}}$ given by

$$
R^{\prime} \stackrel{\pi_{i j}}{\longrightarrow} R^{\prime}\{1-n\} \stackrel{x_{i}-x_{j}}{\longrightarrow} R^{\prime},
$$

where $\pi_{i j}=x_{i}^{n}+x_{i}^{n-1} x_{j}+\cdots+x_{i} x_{j}^{n-1}+x_{j}^{n}$. The purpose of the grading shift here is to make $C_{n}\left(L_{j}^{i}\right)$ graded in the sense that both $\pi_{i j}$ and $x_{i}-x_{j}$ are homogeneous maps of degree $n+1$.

Also, let $C\left(L_{j}^{i}\right)$ be the matrix factorization $\left(a, x_{i}-x_{j}\right)_{R}$ given by

$$
R \stackrel{a}{\rightarrow} R\{-1,1\} \stackrel{x_{i}-x_{j}}{\longrightarrow} R,
$$

where the grading shift makes it bi-graded in the sense that $a$ and $x_{i}-x_{j}$ are both homogeneous maps of bi-degree $(1,1)$.

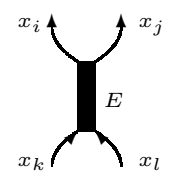

Figure 3 . Wide edge $E$

For a wide edge $E$, let $x_{i}, x_{j}, x_{k}, x_{l}$ be the closest markings to $E$ as depicted in Figure 3. (It is possible that $x_{i}=x_{k}$ or $x_{j}=x_{l}$.) Let $g$ be the unique two-variable polynomial such that $g(x+y, x y)=x^{n+1}+y^{n+1}$, and

$$
\begin{aligned}
& u_{1}=u_{1}\left(x_{i}, x_{j}, x_{k}, x_{l}\right)=\frac{g\left(x_{i}+x_{j}, x_{i} x_{j}\right)-g\left(x_{k}+x_{l}, x_{i} x_{j}\right)}{x_{i}+x_{j}-x_{k}-x_{l}}, \\
& u_{2}=u_{2}\left(x_{i}, x_{j}, x_{k}, x_{l}\right)=\frac{g\left(x_{k}+x_{l}, x_{i} x_{j}\right)-g\left(x_{k}+x_{l}, x_{k} x_{l}\right)}{x_{i} x_{j}-x_{k} x_{l}} .
\end{aligned}
$$

Note that $u_{1}$ and $u_{2}$ are homogeneous polynomials in $x_{i}, x_{j}, x_{k}$ and $x_{l}$ of degrees $2 n$ and $2 n-2$, respectively.

Define $C_{n}(E)$ to be the matrix factorization

$$
\left(\begin{array}{cc}
u_{1} & x_{i}+x_{j}-x_{k}-x_{l} \\
u_{2} & x_{i} x_{j}-x_{k} x_{l}
\end{array}\right)_{R^{\prime}}\{-1\}
$$

which is the tensor product of the matrix factorizations

$$
R^{\prime}\{-1\} \stackrel{u_{1}}{\longrightarrow} R^{\prime}\{-n\} \stackrel{x_{i}+x_{j}-x_{k}-x_{l}}{\longrightarrow} R^{\prime}\{-1\}
$$

and

$$
R^{\prime} \stackrel{u_{2}}{\longrightarrow} R^{\prime}\{3-n\} \stackrel{x_{i} x_{j}-x_{k} x_{l}}{\longrightarrow} R^{\prime} .
$$

Again, the grading shifts here make $C_{n}(E)$ graded in the sense that both differential maps are homogeneous maps of degree $n+1$.

Also define $C(E)$ to be the matrix factorization

$$
\left(\begin{array}{cc}
a & x_{i}+x_{j}-x_{k}-x_{l} \\
0 & x_{i} x_{j}-x_{k} x_{l}
\end{array}\right)_{R},
$$


which is the tensor product of the matrix factorizations

$$
R \stackrel{a}{\rightarrow} R\{-1,1\} \stackrel{x_{i}+x_{j}-x_{k}-x_{l}}{\longrightarrow} R
$$

and

$$
R \stackrel{0}{\rightarrow} R\{-1,3\} \stackrel{x_{i} x_{j}-x_{k} x_{l}}{\longrightarrow} R .
$$

Again, the grading shifts here make $C(E)$ bi-graded in the sense that both differential maps are homogeneous maps of degree $(1,1)$.

Finally, we define

$$
C_{n}(\Gamma)=\left(\bigotimes_{L_{j}^{i}} C_{n}\left(L_{j}^{i}\right)\right) \otimes\left(\bigotimes_{E} C_{n}(E)\right)
$$

and

$$
C(\Gamma)=\left(\bigotimes_{L_{j}^{i}} C\left(L_{j}^{i}\right)\right) \otimes\left(\bigotimes_{E} C(E)\right)
$$

where $L_{j}^{i}$ runs through all the (regular) oriented arcs starting and ending at marked points with no marked interior points, and $E$ runs through all wide edges.

If $\Gamma$ is closed, i.e., has no open end points, then $C_{n}(\Gamma)$ and $C(\Gamma)$ are graded cyclic chain complexes. We denote by $H_{n}(\Gamma)$ and $H(\Gamma)$ their cohomologies. These cohomologies inherit the gradings of $C_{n}(\Gamma)$ and $C(\Gamma)$. Note that the $\mathbb{Z}_{2}$-grading from the cyclic complex structure is trivial since all the non-vanish cohomologies concentrate on one of the two degrees. The cohomologies $H_{n}(\Gamma)$ and $H(\Gamma)$ do not depend on the choice of marked points.

If $\Gamma$ has open end points, let $x_{i_{1}}, \cdots, x_{i_{q}}$ be the markings of these open end points. Then $C_{n}(\Gamma)$ is a matrix factorization of potential $\sum_{r=1}^{q} \pm x_{i_{r}}^{n+1}$, and $C(\Gamma)$ is a factorization of potential $\sum_{r=1}^{q} \pm a x_{i_{r}}$, where the sign is "+" if the corresponding open end point is an exit, and is "-" if otherwise. Let $I^{\prime}$ and $I$ be the ideals generated by $\left\{x_{i_{1}}, \cdots, x_{i_{q}}\right\}$ in $R^{\prime}$ and $R$, respectively. Then $C_{n}(\Gamma) / I^{\prime} C_{n}(\Gamma)$ and $C(\Gamma) / I C(\Gamma)$ are graded cyclic chain complexes. Again, we denote by $H_{n}(\Gamma)$ and $H(\Gamma)$ their cohomologies, which have the gradings inherited from $C_{n}(\Gamma)$ and $C(\Gamma)$. As before, the cohomologies $H_{n}(\Gamma)$ and $H(\Gamma)$ do not depend on the choice of marked points.

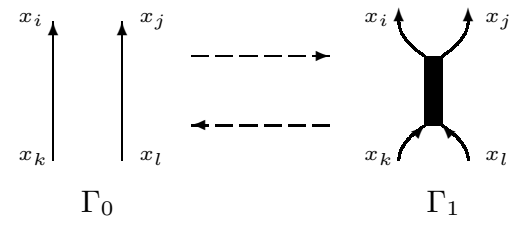

FiguRE $4 . \Gamma_{0}$ and $\Gamma_{1}$

2.5. Khovanov-Rozansky cohomologies of a knot. Let $\Gamma_{0}$ and $\Gamma_{1}$ be the two planar diagrams depicted in Figure 4. Then the matrix factorization $C_{n}\left(\Gamma_{0}\right)$ is

$$
\left(\begin{array}{cc}
\pi_{i k} & x_{i}-x_{k} \\
\pi_{j l} & x_{j}-x_{l}
\end{array}\right)_{R^{\prime}}
$$

Explicitly, this is

$$
\left[\begin{array}{c}
R^{\prime} \\
R^{\prime}\{2-2 n\}
\end{array}\right] \stackrel{P_{0}^{(n)}}{\longrightarrow}\left[\begin{array}{l}
R^{\prime}\{1-n\} \\
R^{\prime}\{1-n\}
\end{array}\right] \stackrel{P_{1}^{(n)}}{\longrightarrow}\left[\begin{array}{c}
R^{\prime} \\
R^{\prime}\{2-2 n\}
\end{array}\right],
$$


where

$$
P_{0}^{(n)}=\left(\begin{array}{cc}
\pi_{i k} & x_{j}-x_{l} \\
\pi_{j l} & -x_{i}+x_{k}
\end{array}\right), \quad P_{1}^{(n)}=\left(\begin{array}{cc}
x_{i}-x_{k} & x_{j}-x_{l} \\
\pi_{j l} & -\pi_{i k}
\end{array}\right) .
$$

The matrix factorization $C_{n}\left(\Gamma_{1}\right)$ is

Explicitly, this is

$$
\left(\begin{array}{cc}
u_{1} & x_{i}+x_{j}-x_{k}-x_{l} \\
u_{2} & x_{i} x_{j}-x_{k} x_{l}
\end{array}\right)_{R^{\prime}}\{-1\}
$$

$$
\left[\begin{array}{c}
R^{\prime}\{-1\} \\
R^{\prime}\{3-2 n\}
\end{array}\right] \stackrel{Q_{0}^{(n)}}{\longrightarrow}\left[\begin{array}{c}
R^{\prime}\{-n\} \\
R^{\prime}\{2-n\}
\end{array}\right] \stackrel{Q_{1}^{(n)}}{\longrightarrow}\left[\begin{array}{c}
R^{\prime}\{-1\} \\
R^{\prime}\{3-2 n\}
\end{array}\right]
$$

where

$$
\begin{aligned}
Q_{0}^{(n)} & =\left(\begin{array}{cc}
u_{1} & x_{i} x_{j}-x_{k} x_{l} \\
u_{2} & -x_{i}-x_{j}+x_{k}+x_{l}
\end{array}\right), \\
Q_{1}^{(n)} & =\left(\begin{array}{cc}
x_{i}+x_{j}-x_{k}-x_{l} & x_{i} x_{j}-x_{k} x_{l} \\
u_{2} & -u_{1}
\end{array}\right) .
\end{aligned}
$$

Define $\chi_{0}^{(n)}: C_{n}\left(\Gamma_{0}\right) \rightarrow C_{n}\left(\Gamma_{1}\right)$ by the matrices

$$
U_{0}^{(n)}=\left(\begin{array}{cc}
x_{k}-x_{j} & 0 \\
a_{1} & 1
\end{array}\right), \quad U_{1}^{(n)}=\left(\begin{array}{cc}
x_{k} & -x_{j} \\
-1 & 1
\end{array}\right)
$$

and $\chi_{1}^{(n)}: C_{n}\left(\Gamma_{1}\right) \rightarrow C_{n}\left(\Gamma_{0}\right)$ by the matrices

$$
V_{0}^{(n)}=\left(\begin{array}{cc}
1 & 0 \\
-a_{1} & x_{k}-x_{j}
\end{array}\right), \quad V_{1}^{(n)}=\left(\begin{array}{cc}
1 & x_{j} \\
1 & x_{k}
\end{array}\right),
$$

where $a_{1}=-u_{2}+\left(u_{1}+x_{i} u_{2}-\pi_{j l}\right) /\left(x_{i}-x_{k}\right)$. These are homomorphisms of matrix factorizations of degree 1, i.e., these commute with the differential maps and raise the quantum grading by 1 . So $\chi_{0}^{(n)}$ and $\chi_{1}^{(n)}$ induce homomorphisms of cohomologies. One can check that

$$
\chi_{1}^{(n)} \chi_{0}^{(n)}=\left(x_{k}-x_{j}\right) \operatorname{id}_{C_{n}\left(\Gamma_{0}\right)}, \quad \chi_{0}^{(n)} \chi_{1}^{(n)}=\left(x_{k}-x_{j}\right) \operatorname{id}_{C_{n}\left(\Gamma_{1}\right)} .
$$

There is a similar construction for $C\left(\Gamma_{0}\right)$ and $C\left(\Gamma_{1}\right)$. Explicitly, the matrix factorization $C\left(\Gamma_{0}\right)$ is

where

$$
\left[\begin{array}{c}
R \\
R\{-2,2\}
\end{array}\right] \stackrel{P_{0}}{\longrightarrow}\left[\begin{array}{c}
R\{-1,1\} \\
R\{-1,1\}
\end{array}\right] \stackrel{P_{1}}{\longrightarrow}\left[\begin{array}{c}
R \\
R\{-2,2\}
\end{array}\right],
$$

$$
P_{0}=\left(\begin{array}{cc}
a & x_{j}-x_{l} \\
a & -x_{i}+x_{k}
\end{array}\right), \quad P_{1}=\left(\begin{array}{cc}
x_{i}-x_{k} & x_{j}-x_{l} \\
a & -a
\end{array}\right),
$$

and the matrix factorization $C\left(\Gamma_{1}\right)$ is

$$
\left[\begin{array}{c}
R \\
R\{-2,4\}
\end{array}\right] \stackrel{Q_{0}}{\longrightarrow}\left[\begin{array}{l}
R\{-1,1\} \\
R\{-1,3\}
\end{array}\right] \stackrel{Q_{1}}{\longrightarrow}\left[\begin{array}{c}
R \\
R\{-2,4\}
\end{array}\right]
$$

where

$$
Q_{0}=\left(\begin{array}{cc}
a & x_{i} x_{j}-x_{k} x_{l} \\
0 & -x_{i}-x_{j}+x_{k}+x_{l}
\end{array}\right), \quad Q_{1}=\left(\begin{array}{cc}
x_{i}+x_{j}-x_{k}-x_{l} & x_{i} x_{j}-x_{k} x_{l} \\
0 & -a
\end{array}\right) .
$$

Define $\chi_{0}: C\left(\Gamma_{0}\right) \rightarrow C\left(\Gamma_{1}\right)$ by the matrices

$$
U_{0}=\left(\begin{array}{cc}
x_{k}-x_{j} & 0 \\
0 & 1
\end{array}\right), \quad U_{1}=\left(\begin{array}{cc}
x_{k} & -x_{j} \\
-1 & 1
\end{array}\right)
$$


and $\chi_{1}: C\left(\Gamma_{1}\right) \rightarrow C\left(\Gamma_{0}\right)$ by the matrices

$$
V_{0}=\left(\begin{array}{cc}
1 & 0 \\
0 & x_{k}-x_{j}
\end{array}\right), \quad V_{1}^{(n)}=\left(\begin{array}{cc}
1 & x_{j} \\
1 & x_{k}
\end{array}\right) .
$$

These are homomorphisms of matrix factorizations and induce homomorphisms of cohomologies. One can check that $\chi_{0}$ and $\chi_{1}$ are of bi-degrees $(0,2)$ and $(0,0)$, respectively, and

$$
\chi_{1} \chi_{0}=\left(x_{k}-x_{j}\right) \operatorname{id}_{C\left(\Gamma_{0}\right)}, \quad \chi_{0} \chi_{1}=\left(x_{k}-x_{j}\right) \operatorname{id}_{C\left(\Gamma_{1}\right)} .
$$

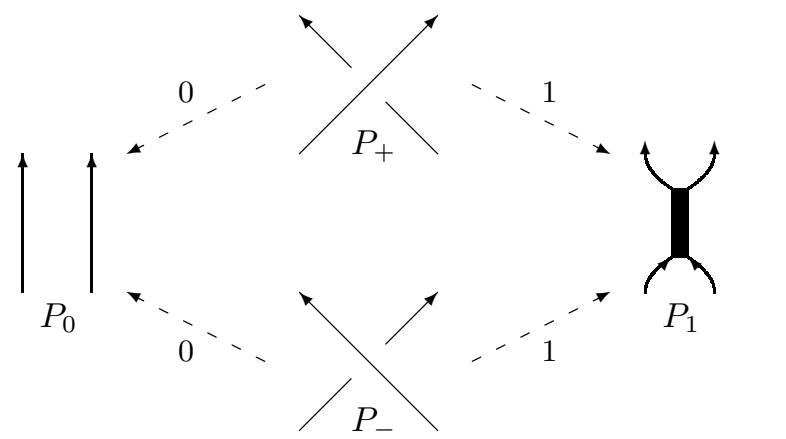

FiguRE 5. Resolutions of a crossing

Let $D$ be an oriented link diagram. We put markings $\left\{x_{1}, \cdots, x_{m}\right\}$ on $D$ so that none of the crossings is marked, and each arc between two crossings has a marked point. For each crossing in $D$, there are two ways to resolve it as shown in Figure 5. A resolution of $D$ is a planar diagram obtained from $D$ by resolving all the crossings of $D$. Together with the markings inherited from $D$, each resolution of $D$ is a planar diagram satisfying the conditions in Subsection 2.3.

For an arc $L_{j}^{i}$ from $x_{j}$ to $x_{i}$ that contains no crossings and no other marked points, define $C_{n}\left(L_{j}^{i}\right)$ as above and consider it as the chain complex

$$
0 \rightarrow \underbrace{C_{n}\left(L_{j}^{i}\right)}_{0} \rightarrow 0
$$

where $C_{n}\left(L_{j}^{i}\right)$ has cohomological degree 0 .

For a positive crossing $P_{+}$in $D$, let $P_{0}$ and $P_{1}$ be the two diagrams depicted in Figure 5, Define $C_{n}\left(P_{+}\right)$to be the chain complex

$$
0 \rightarrow \underbrace{C_{n}\left(P_{1}\right)\{n\}}_{-1} \stackrel{\chi_{1}^{(n)}}{\longrightarrow} \underbrace{C_{n}\left(P_{0}\right)\{n-1\}}_{0} \rightarrow 0,
$$

where $C_{n}\left(P_{0}\right)\{n-1\}$ has cohomological degree 0 , and $C_{n}\left(P_{1}\right)\{n\}$ has cohomological degree -1 .

For a negative crossing $P_{-}$in $D$, define $C_{n}\left(P_{+}\right)$to be the chain complex

$$
0 \rightarrow \underbrace{C_{n}\left(P_{0}\right)\{1-n\}}_{0} \stackrel{\chi_{0}^{(n)}}{\longrightarrow} \underbrace{C_{n}\left(P_{1}\right)\{-n\}}_{1} \rightarrow 0,
$$

where $C_{n}\left(P_{0}\right)\{1-n\}$ has cohomological degree 0 , and $C_{n}\left(P_{1}\right)\{-n\}$ has cohomological degree 1. 
Now define $C_{n}(D)$ to be the chain complex

$$
C_{n}(D)=\left(\bigotimes_{L_{j}^{i}} C_{n}\left(L_{j}^{i}\right)\right) \otimes\left(\bigotimes_{P} C_{n}(P)\right)
$$

where $L_{j}^{i}$ runs through all arcs in $D$ starting and ending in marked points that contain no crossings and no other marked points, and $P$ runs through all the crossings of $D$.

Remark 2.1. (1) Disregarding the chain complex structure on $C_{n}(D)$, we have

$$
C_{n}(D)=\bigoplus_{\Gamma} C_{n}(\Gamma)\left\{p_{\Gamma}\right\}
$$

where $\Gamma$ runs through all resolutions of $D, p_{\Gamma} \in \mathbb{Z}$. For a given $\Gamma$, all elements of $C_{n}(\Gamma)$ have the same cohomological degree.

(2) There are two differential maps on $C_{n}(D)$. One comes from the matrix factorizations $C_{n}(\Gamma)$, which we denote by $d_{m f}$. The other comes from the above construction, which we denote by $d_{\chi}$. These two differentials commute.

(3) $C_{n}(D)$ is $\mathbb{Z} \oplus \mathbb{Z} \oplus \mathbb{Z}_{2}$-graded, where the first $\mathbb{Z}$-grading is the cohomological grading, the second is inherited from the $\mathbb{Z}$-grading of $C_{n}(\Gamma)\left\{p_{\Gamma}\right\}$, and is called the quantum grading, and the $\mathbb{Z}_{2}$-grading comes from the cyclic chain complex structure of $C_{n}(\Gamma)$. Note that $d_{\chi}$ preserves the quantum grading.

$d_{\chi}$ induces a differential map on the cohomology $H\left(C_{n}(D), d_{m f}\right)$, which we still denote by $d_{\chi}$. Define $H_{n}(D)$ to be the cohomology

$$
H_{n}(D)=H\left(H\left(C_{n}(D), d_{m f}\right), d_{\chi}\right) .
$$

$H_{n}(D)$ inherits the $\mathbb{Z} \oplus \mathbb{Z}$-gradings from $C_{n}(D)$. The induced $\mathbb{Z}_{2}$-grading on $H_{n}(D)$ is trivial since all the non-zero elements concentrate on the degree equal to the $\mathbb{Z}_{2^{-}}$ number of components of the link represented by $D$.

M. Khovanov and L. Rozansky proved in [7] that the $\mathbb{Z} \oplus \mathbb{Z}$-graded isomorphism type of $H_{n}(D)$ is invariant under Reidemeister moves, and, therefore, is a link invariant. They also showed that, up to multiplication by a non-zero scalar, the homomorphism of $H_{n}$ induced by a link cobordism is independent of the movie representation. In particular, this implies that the $H_{n}$ theory gives bona fide homology groups, not just isomorphism classes.

The homology $H(D)$ is defined similarly. To $L_{j}^{i}, P_{+}$and $P_{-}$, we assign the chain complexes

$$
\begin{gathered}
C\left(L_{j}^{i}\right)=[0 \rightarrow \underbrace{C\left(L_{j}^{i}\right)}_{0} \rightarrow 0], \\
C\left(P_{+}\right)=[0 \rightarrow \underbrace{C\left(P_{1}\right)\{0,-2\}}_{0} \stackrel{\chi_{1}}{\longrightarrow} \underbrace{C\left(P_{0}\right)\{0,-2\}}_{1} \rightarrow 0], \\
C\left(P_{-}\right)=[0 \rightarrow \underbrace{C\left(P_{0}\right)\{0,2\}}_{-1} \stackrel{\chi_{0}}{\longrightarrow} \underbrace{C\left(P_{1}\right)}_{0} \rightarrow 0],
\end{gathered}
$$

where the number below each term indicates its cohomological degree. Then define

$$
C(D)=\left(\bigotimes_{L_{j}^{i}} C\left(L_{j}^{i}\right)\right) \otimes\left(\bigotimes_{P} C(P)\right)
$$


where $L_{j}^{i}$ runs through all arcs in $D$ starting and ending in marked points that contain no crossings and no other marked points, and $P$ runs through all the crossings of $D$. Again, we have

$$
C(D)=\bigoplus_{\Gamma} C(\Gamma)\left\{0, q_{\Gamma}\right\}
$$

where $\Gamma$ runs through all resolutions of $D, q_{\Gamma} \in \mathbb{Z}$. Again, there are two differential maps: $d_{m f}$ from the matrix factorization structure, and $d_{\chi}$ from the above chain complex construction. These commute with each other, and $d_{\chi}$ preserves the two quantum gradings. We define

$$
H(D)=H\left(H\left(C(D), d_{m f}\right), d_{\chi}\right) .
$$

$H(D)$ inherits the $\mathbb{Z} \oplus \mathbb{Z} \oplus \mathbb{Z}$-grading of $C(D)$, i.e. the cohomological grading, the quantum $a$-grading and the quantum $x$-grading. M. Khovanov and L. Rozansky proved in [7] that the $\mathbb{Z} \oplus \mathbb{Z} \oplus \mathbb{Z}$-graded isomorphism type of $H(D)$ is invariant under Markov moves of braids up to overall shift of grading. Thus, any two braids that represent the same link have isomorphic $H$-invariant. Given a braid $B$ with $b$ strands and writhe $w$, define

$$
\hat{H}(B)=H(B)\left\{\frac{w+b}{2}, \frac{w+b}{2}\right\}\left[-\frac{w+b}{2}\right],
$$

where $\left[-\frac{w+b}{2}\right]$ means shifting the homological grading by $-\frac{w+b}{2}$. From the construction in [8], it's easy to see that the $\frac{1}{2} \mathbb{Z} \oplus \frac{1}{2} \mathbb{Z} \oplus \frac{1}{2} \mathbb{Z}$-graded isomorphism type of $\hat{H}(B)$ is honestly invariant under Markov moves of braids.

2.6. Graded Euler characteristics. We normalize the HOMFLY polynomial $P$ by the skein relation

$$
\left\{\begin{array}{l}
x P\left(L_{-}\right)-x^{-1} P\left(L_{+}\right)=y P\left(L_{0}\right), \\
P(\text { unknot })=\frac{x-x^{-1}}{y},
\end{array}\right.
$$

where the diagrams of $L_{+}, L_{-}$and $L_{0}$ are identical except at one crossing, where they look like $P_{+}, P_{-}$and $P_{0}$ in Figure 5, respectively.

For a link $L$, consider the graded Euler characteristic

$$
F_{n}(L)=\sum_{j, k}(-1)^{j} t^{k} \operatorname{dim} H_{n}^{j, k}(L) .
$$

From [7, we know that $F_{n}$ is characterized by the following skein relation:

$$
\left\{\begin{array}{l}
t^{n} F_{n}\left(L_{-}\right)-t^{-n} F_{n}\left(L_{+}\right)=\left(t-t^{-1}\right) F_{n}\left(L_{0}\right) \\
F_{n} \text { (unknot) }=\frac{t^{n}-t^{-n}}{t-t^{-1}}
\end{array}\right.
$$

So

$$
F_{n}(L)(t)=P(L)\left(t^{n}, t-t^{-1}\right) .
$$

Next, consider the graded Euler characteristic

$$
\hat{F}(L)=\sum_{j, k, l}(-1)^{j} t^{k} q^{l} \operatorname{dim} \hat{H}^{j, k, l}(L) .
$$

From [8, we know that $\hat{F}$ is characterized by the following skein relation:

$$
\left\{\begin{array}{l}
-q^{-1}(-t q)^{\frac{1}{2}} \hat{F}\left(L_{-}\right)+q(-t q)^{-\frac{1}{2}} \hat{F}\left(L_{+}\right)=\left(q-q^{-1}\right) \hat{F}\left(L_{0}\right), \\
\hat{F}(\text { unknot })=-\frac{\sqrt{-1} t^{-\frac{1}{2}} q^{\frac{1}{2}}}{q-q^{-1}}
\end{array}\right.
$$


where $L_{+}, L_{-}$and $L_{0}$ are represented by braid diagrams that are identical except at one crossing, where they look like $P_{+}, P_{-}$and $P_{0}$ in Figure 5 , respectively.

Change the variables by

$$
\left\{\begin{array}{l}
x=-q^{-1}(-t q)^{\frac{1}{2}} \\
y=q-q^{-1}
\end{array}\right.
$$

Then, in these new variables, we have

$$
\left\{\begin{array}{l}
x \hat{F}\left(L_{-}\right)-x^{-1} \hat{F}\left(L_{+}\right)=y \hat{F}\left(L_{0}\right) \\
\hat{F}(\text { unknot })=-x^{-1} y^{-1}
\end{array}\right.
$$

Compare these normalizations with those used in 4, 9. It's clear that Theorem 1.1 and Corollary 1.3 imply the corresponding results in 4, 9]. In particular, the two upper bounds of the self-linking number in Theorem 1.4 are sharper than the bound given by the HOMFLY polynomial, which is implicitly proven in 4, 9] (cf. [3, [5]).

\section{RESOLVED BRAIDS}

For positive integers $b, i$ with $1 \leq i \leq b-1$, let $\tau_{i}$ be the diagram depicted in Figure 6. That is, from left to right, $\tau_{i}$ consists of $i-1$ upward vertical regular edges, then a vertical wide edge with two regular edges entering through the bottom and two regular edges exiting through the top, and then $b-i-1$ more upward vertical regular edges.

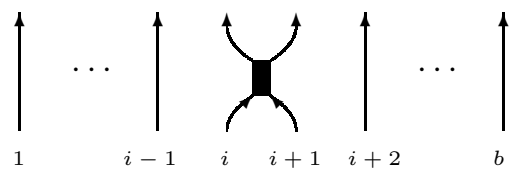

FiguRE 6. $\tau_{i}$

We use the word $\tau_{i_{1}} \cdots \tau_{i_{m}}$ to represent the planar graph formed by stacking the graphs $\tau_{i_{1}}, \cdots, \tau_{i_{m}}$ together vertically from bottom to top with the top end points of $\tau_{i_{l}}$ identified with the corresponding bottom end points of $\tau_{i_{l+1}}$. The symbol $\underline{\tau_{i_{1}} \cdots \tau_{i_{m}}}$ represents the closed graph obtained from $\tau_{i_{1}} \cdots \tau_{i_{m}}$ by attaching a disjoint regular edge from each end point on the top to the corresponding end point at the bottom. We use the convention that the empty word $\phi$ represents $b$ vertical upward regular edges, and, therefore, $\underline{\phi}$ represents $b$ concentric circles. We call $\tau_{i_{1}} \cdots \tau_{i_{m}}$ a resolved braid, and $\underline{\tau_{i_{1}} \cdots \tau_{i_{m}}}$ a resolved closed braid. See Figure 7 for examples of such graphs. There are two obvious types of isotopies:

(I) If $|i-j|>1$, then $\tau_{i} \tau_{j}$ is isotopic to $\tau_{j} \tau_{i}$.

(II) If $\mu$ and $\nu$ are two words in $\tau_{1}, \cdots, \tau_{b-1}$, then $\underline{\mu \nu}$ is isotopic to $\underline{\nu \mu}$.

We prove inequality (1.1) in Theorem 1.1 first.

For a word $\mu$ in $\tau_{1}, \cdots, \tau_{b-1}$, consider the $\mathbb{Z} \oplus \mathbb{Z}$-graded cohomology $H(\underline{\mu})=$ $H\left(C(\underline{\mu}), d_{m f}\right)$. Denote by $H^{k, l}(\underline{\mu})$ the subspace of $H(\underline{\mu})$ consisting of homogeneous elements of $\mathbb{Z} \oplus \mathbb{Z}$ grading $(k, l)$, where $k$ is the quantum $a$-grading, and $l$ is the quantum $x$-grading. Define

$$
\begin{aligned}
g_{\max }(\underline{\mu}) & =\max \left\{k \mid H^{k, l}(\underline{\mu}) \neq 0 \text { for some } l\right\}, \\
g_{\min }(\underline{\mu}) & =\min \left\{k \mid H^{k, l}(\underline{\mu}) \neq 0 \text { for some } l\right\} .
\end{aligned}
$$




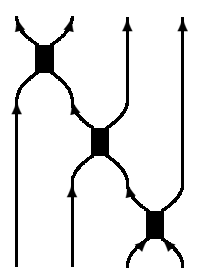

$\tau_{3} \tau_{2} \tau_{1}$

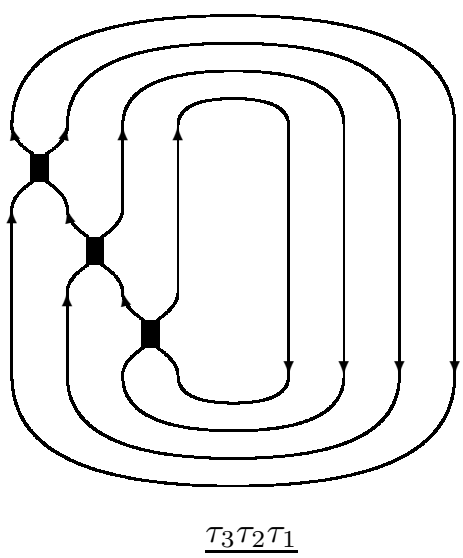

for $b=4$.

In order to calculate $H(\underline{\mu})$, we need to put marks on regular edges of $\underline{\mu}$. But, as long as there is at least one mark on each regular edge, the result is independent of the choice of the marks.

Lemma 3.1. For the empty word $\phi$ (with $b$ strands), we have $g_{\max }(\underline{\phi})=-1$ and $g_{\min }(\underline{\phi})=-b$.

Proof. $\underline{\phi}$ represents $b$ concentric circles. We order the circles, and put the marking $x_{i}$ on the $i$-th circle. The cyclic Koszul complex $C(\underline{\phi})$ is given over $R=$ $\mathbb{Q}\left[a, x_{1}, \cdots, x_{b}\right]$ by

$$
\left(\begin{array}{cc}
a & 0 \\
\vdots & \vdots \\
a & 0
\end{array}\right),
$$

where there are $b$ rows. After $b-1$ elementary transformations of rows (cf. subsection 2.1 of $[8]$ ), we see that it is isomorphic to

$$
\left(\begin{array}{cc}
a & 0 \\
0 & 0 \\
\vdots & \vdots \\
0 & 0
\end{array}\right),
$$

which is isomorphic to the chain complex

$$
C \otimes_{\mathbb{Q}} D^{\otimes(b-1)} \otimes_{\mathbb{Q}} \mathbb{Q}\left[x_{1}, \cdots, x_{b}\right],
$$

where $C$ is the chain complex

$$
0 \rightarrow \mathbb{Q}[a] \stackrel{a}{\rightarrow} \mathbb{Q}[a]\{-1,1\} \rightarrow 0
$$

and $D$ is the chain complex

$$
0 \rightarrow \mathbb{Q} \stackrel{0}{\rightarrow} \mathbb{Q}\{-1,1\} \rightarrow 0 .
$$


Then, by the Künneth Theorem and the Universal Coefficient Theorem, it's easy to check that

$$
\begin{aligned}
H(\underline{\phi}) & =H\left(C \otimes_{\mathbb{Q}} D^{\otimes(b-1)} \otimes_{\mathbb{Q}} \mathbb{Q}\left[x_{1}, \cdots, x_{b}\right]\right) \\
& =H(C) \otimes_{\mathbb{Q}} H\left(D^{\otimes(b-1)}\right) \otimes_{\mathbb{Q}} \mathbb{Q}\left[x_{1}, \cdots, x_{b}\right] \\
& =\mathbb{Q}\{-1,1\} \otimes_{\mathbb{Q}} D^{\otimes(b-1)} \otimes_{\mathbb{Q}} \mathbb{Q}\left[x_{1}, \cdots, x_{b}\right] .
\end{aligned}
$$

From the definition of $D$, one can see that the maximal and minimal quantum $a$-gradings of the right hand side are -1 and $-b$. This proves the lemma.

Lemma 3.2. If $\mu=\tau_{i_{1}} \cdots \tau_{i_{m}}$ is a word in $\tau_{1}, \cdots, \tau_{b-1}$ with $i_{p}<i$ for $1 \leq p \leq m$, and $\mu^{\prime}=\mu \tau_{i}$, then $g_{\max }(\underline{\mu})=g_{\max }\left(\underline{\mu^{\prime}}\right)$, and $g_{\min }(\underline{\mu})=g_{\min }\left(\underline{\mu^{\prime}}\right)$.

Proof. The only difference between $\underline{\mu}$ and $\underline{\mu}$ occurs in the part depicted in Figure 8 .
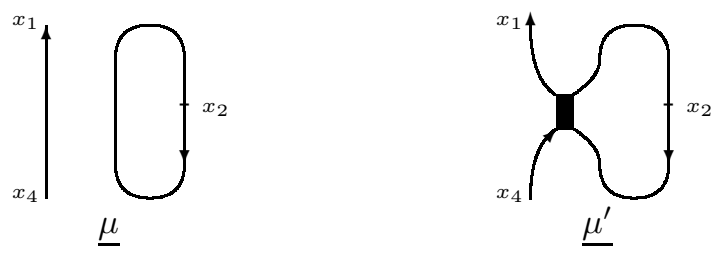

FIGURE 8. $\underline{\mu}$ and $\underline{\mu^{\prime}}$

The local matrix factorization of the part of $\underline{\mu}$ in Figure 8 is

$$
\left(\begin{array}{cc}
a & x_{1}-x_{4} \\
a & 0
\end{array}\right) \text {. }
$$

After an elementary transformation, it becomes

$$
\left(\begin{array}{cc}
a & x_{1}-x_{4} \\
0 & 0
\end{array}\right)
$$

Write $R=\mathbb{Q}\left[a, x_{1}, x_{2}, x_{4}\right]$. Explicitly, this matrix factorization is

$$
R \oplus R\{-2,2\} \rightarrow R\{-1,1\} \oplus R\{-1,1\} \rightarrow R \oplus R\{-2,2\}
$$

where

$$
d^{0}=\left(\begin{array}{cc}
a & 0 \\
0 & -x_{1}+x_{4}
\end{array}\right) \text { and } d^{1}=\left(\begin{array}{cc}
x_{1}-x_{4} & 0 \\
0 & -a
\end{array}\right) .
$$

The local matrix factorization of the part of $\underline{\mu}^{\prime}$ in Figure 8 is

$$
\left(\begin{array}{cc}
a & x_{1}-x_{4} \\
0 & 0
\end{array}\right) \text {. }
$$

Explicitly, this matrix factorization is

$$
R \oplus R\{-2,4\} \rightarrow R\{-1,1\} \oplus R\{-1,3\} \rightarrow R \oplus R\{-2,4\},
$$

where

$$
d^{0}=\left(\begin{array}{cc}
a & 0 \\
0 & -x_{1}+x_{4}
\end{array}\right) \text { and } d^{1}=\left(\begin{array}{cc}
x_{1}-x_{4} & 0 \\
0 & -a
\end{array}\right) .
$$

Let $f$ be the map between the two matrix factorizations given by

$$
\left(\begin{array}{ll}
1 & 0 \\
0 & 1
\end{array}\right): R \oplus R\{-2,2\} \rightarrow R \oplus R\{-2,4\},
$$


and

$$
\left(\begin{array}{ll}
1 & 0 \\
0 & 1
\end{array}\right): R\{-1,1\} \oplus R\{-1,1\} \rightarrow R\{-1,1\} \oplus R\{-1,3\}
$$

It's clear that $f$ is an isomorphism of matrix factorizations that preserves the quantum $a$-grading (but not the $x$-grading). After tensoring $f$ with the identity maps of other local matrix factorizations of $\mu$ (and $\mu^{\prime}$ ), we get an isomorphism between these matrix factorizations that preserves the $a$-grading. This proves the lemma.

Lemma 3.3. If $\nu$ is a word in $\tau_{1}, \cdots, \tau_{b-1}, \mu=\nu \tau_{i}$ and $\mu^{\prime}=\nu \tau_{i} \tau_{i}$, then $g_{\max }(\underline{\mu})=$ $g_{\max }\left(\underline{\mu^{\prime}}\right)$, and $g_{\min }(\underline{\mu})=g_{\min }\left(\underline{\mu^{\prime}}\right)$.

Proof. (Follow Proposition 30 of [7].) The only difference between $\underline{\mu}$ and $\underline{\mu^{\prime}}$ occurs in the part depicted in Figure 9 .
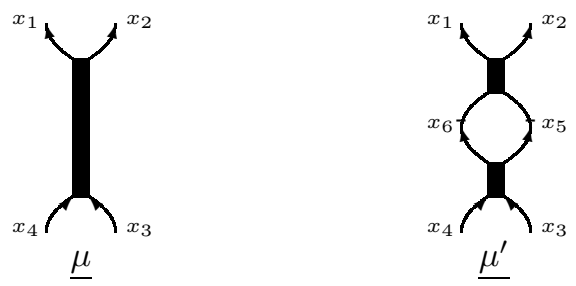

FIGURE 9. $\underline{\mu}$ and $\underline{\mu^{\prime}}$

Write $R=\mathbb{Q}\left[a, x_{1}, \cdots, x_{6}\right]$. Let $s_{1}=x_{5}+x_{6}, s_{2}=x_{5} x_{6}$, and $\check{R}=\mathbb{Q}\left[a, x_{1}, x_{2}, x_{3}\right.$, $\left.x_{4}, s_{1}, s_{2}\right]$. Then $R=\check{R} \oplus \check{R}\{0,2\}$.

The local matrix factorization $M^{\prime}$ of the part of $\mu^{\prime}$ in Figure 8 is

$$
\left(\begin{array}{cc}
a & x_{1}+x_{2}-s_{1} \\
0 & x_{1} x_{2}-s_{2} \\
a & s_{1}-x_{3}-x_{4} \\
0 & s_{2}-x_{3} x_{4}
\end{array}\right)_{R} .
$$

Let $\check{M}^{\prime}$ be the matrix factorization over $\check{R}$ given by the same sequence. Then $M^{\prime}=\check{M}^{\prime} \oplus \check{M}^{\prime}\{0,2\}$. After elementary transformations, $\check{M}^{\prime}$ is isomorphic to

$$
\left(\begin{array}{cc}
a & x_{1}+x_{2}-x_{3}-x_{4} \\
0 & x_{1} x_{2}-x_{3} x_{4} \\
0 & s_{1}-x_{3}-x_{4} \\
0 & s_{2}-x_{3} x_{4}
\end{array}\right)_{\check{R}} .
$$

We then exclude the variables $s_{1}, s_{2}$ and remove the last two rows from the above sequence. This does not change the chain homotopy type of the matrix factorization (cf. Proposition 3 of [8]). The result of this operation is a matrix factorization over $\mathbb{Q}\left[a, x_{1}, x_{2}, x_{3}, x_{4}\right]$ given by the sequence

$$
\left(\begin{array}{cc}
a & x_{1}+x_{2}-x_{3}-x_{4} \\
0 & x_{1} x_{2}-x_{3} x_{4}
\end{array}\right)
$$

which is exactly the local matrix factorization of the part of $\underline{\mu}$ depicted in Figure 9. This implies that $H\left(\underline{\mu^{\prime}}\right)=H(\underline{\mu}) \oplus H(\underline{\mu})\{0,2\}$, and the lemma follows.

Lemma 3.4. If $\nu$ is a word in $\tau_{1}, \cdots, \tau_{b-1}, \mu_{1}=\nu \tau_{i-1} \tau_{i} \tau_{i-1}, \mu_{2}=\nu \tau_{i}$, and $\mu_{3}=$ $\nu \tau_{i} \tau_{i-1} \tau_{i}$, then $g_{\max }\left(\underline{\mu_{3}}\right) \leq \max \left\{g_{\max }\left(\underline{\mu_{1}}\right), g_{\max }\left(\underline{\mu_{2}}\right)\right\}, g_{\min }\left(\underline{\mu_{3}}\right) \geq \min \left\{g_{\min }\left(\underline{\mu_{1}}\right)\right.$, $\left.g_{\min }\left(\underline{\mu_{2}}\right)\right\}$. 
Proof. Let $\mu_{4}=\nu \tau_{i-1}$. The only difference between $\underline{\mu_{1}}, \underline{\mu_{2}}, \underline{\mu_{3}}$ and $\underline{\mu_{4}}$ occurs in the part depicted in Figure 10 .

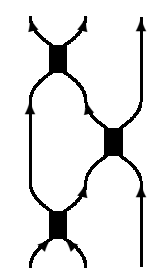

$\underline{\mu_{1}}$

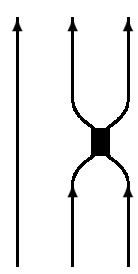

$\underline{\mu_{2}}$

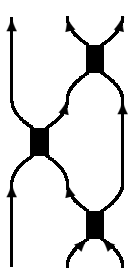

$\underline{\mu_{3}}$

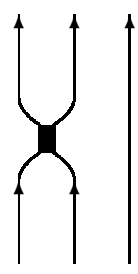

$\underline{\mu_{4}}$

FiguRE 10. $\underline{\mu_{1}}, \underline{\mu_{2}}, \underline{\mu_{3}}$ and $\underline{\mu_{4}}$

By Proposition 7 of 8 ,

$$
H\left(\underline{\mu_{1}}\right) \oplus H\left(\underline{\mu_{2}}\right)\{0,2\}=H\left(\underline{\mu_{3}}\right) \oplus H\left(\underline{\mu_{4}}\right)\{0,2\} .
$$

This implies the lemma.

Lemma 3.5. Let $\mu=\tau_{i_{1}} \cdots \tau_{i_{m}}$ be a word in $\tau_{1}, \cdots, \tau_{b-1}$, such that $m \geq 2$, $i_{1}=i_{m}=i$, and $i_{p}<i$ for $1<p<m$. Then, after possibly finitely many steps of isotopies of type (I), there is a sub-word of $\mu$ of the form $\tau_{j} \tau_{j}$ or $\tau_{j} \tau_{j-1} \tau_{j}$ for some $j \leq i$.

Proof. We induct on the length $m$ of the word $\mu$. When $m=2$, the lemma is trivially true. Assume that the lemma is true for words with the given properties and length $=2, \cdots, m-1$. Now consider $\mu=\tau_{i_{1}} \cdots \tau_{i_{m}}$. If there is none or only one $\tau_{i-1}$ among $\tau_{i_{2}}, \cdots, \tau_{i_{m-1}}$, then the lemma is true for $\mu$ with $j=i$. If there is more than one $\tau_{i-1}$ 's among $\tau_{i_{2}}, \cdots, \tau_{i_{m-1}}$, then there is a subword $\nu=\tau_{j_{1}} \cdots \tau_{j_{l}}$ of $\mu$ with $2 \leq l<m, j_{1}=j_{l}=i-1$, and $j_{q}<i-1$ for $1<q<l$. Thus, by induction, the lemma is true for $\mu$.

Proposition 3.6. If $\mu$ is a word in $\tau_{1}, \cdots, \tau_{b-1}$, then $-b \leq g_{\min }(\underline{\mu}) \leq g_{\max }(\underline{\mu}) \leq$ -1 .

Proof. For a word $\mu=\tau_{i_{1}} \cdots \tau_{i_{m}}$, define the weight $W(\mu)$ of $\mu$ to be

$$
W(\mu)=\sum_{p=1}^{m} i_{p} .
$$

We prove the proposition by induction on the weight of a word. If $W(\mu)=0$, then $\mu$ is the empty word $\phi$, and Lemma 3.1 implies the proposition. Now assume that the proposition is true for all words with weight $<W$, and $\mu=\tau_{i_{1}} \cdots \tau_{i_{m}}$ is a word with weight $W$. Let $i=\max \left\{i_{1}, \cdots, i_{m}\right\}$. If $i$ appears only once among $i_{1}, \cdots, i_{m}$, then, after a possible type (II) isotopy, Lemma 3.2 implies that the proposition is true for $\mu$. If $i$ appears at least twice among $i_{1}, \cdots, i_{m}$, then, after possible type (I) and type (II) isotopies, Lemmas $3.3,3.4$ and 3.5 imply that the proposition is true for $\mu$.

Proof of inequality (1.1) in Theorem 1.1. For a braid $B$ with $b$ strands, consider the Khovanov-Rozansky cohomology $H(B)$, and let

$$
\begin{aligned}
& g_{\text {max }}(B)=\max \left\{k \mid H^{j, k, l}(L) \neq 0 \text { for some } j, l\right\}, \\
& g_{\text {min }}(B)=\min \left\{k \mid H^{j, k, l}(L) \neq 0 \text { for some } j, l\right\} .
\end{aligned}
$$


Note that

and

$$
H(B)=H\left(H\left(C(B), d_{m f}\right), d_{\chi}\right)
$$

$$
H\left(C(B), d_{m f}\right)=\bigoplus_{\Gamma} H(\Gamma)\left\{0, q_{\Gamma}\right\},
$$

where $\Gamma$ runs through all resolutions of $B$, and $q_{\Gamma} \in \mathbb{Z}$. Thus, by Proposition 3.6, we have $-b \leq g_{\min }(B) \leq g_{\max }(B) \leq-1$. But $\hat{H}(B)=H(B)\left\{\frac{w+b}{2}, \frac{w+b}{2}\right\}\left[-\frac{w+b}{2}\right]$. So

$$
\begin{aligned}
& \hat{g}_{\max }(B)=g_{\max }(B)+\frac{w+b}{2}, \\
& \hat{g}_{\min }(B)=g_{\min }(B)+\frac{w+b}{2},
\end{aligned}
$$

and inequality (1.1) follows.

The proof of inequality (1.2) in Theorem 1.1 is similar.

For a word $\mu$ in $\tau_{1}, \cdots, \tau_{b-1}$, consider the $\mathbb{Z}$-graded cohomology $H_{n}(\underline{\mu})=$ $H_{n}\left(C(\underline{\mu}), d_{m f}\right)$. Denote by $H_{n}^{k}(\underline{\mu})$ the subspace of $H_{n}(\underline{\mu})$ consisting of homogeneous elements of quantum grading $k$. Define

$$
\begin{gathered}
g_{\text {max }}^{(n)}(\underline{\mu})=\max \left\{k \mid H_{n}^{k}(\underline{\mu}) \neq 0\right\}, \\
g_{\min }^{(n)}(\underline{\mu})=\min \left\{k \mid H_{n}^{k}(\underline{\mu}) \neq 0\right\} .
\end{gathered}
$$

We have the following conclusions similar to Lemmas 3.1, 3.2, 3.3 and 3.4

Lemma 3.7. (1) Let $\phi$ be the empty word (with $b$ strands). Then

$$
g_{\max }^{(n)}(\underline{\phi})=(n-1) b \text { and } g_{\min }^{(n)}(\underline{\phi})=-(n-1) b .
$$

(2) If $\mu=\tau_{i_{1}} \cdots \tau_{i_{m}}$ is a word in $\tau_{1}, \cdots, \tau_{b-1}$ with $i_{p}<i$ for $1 \leq p \leq m$, and $\mu^{\prime}=\mu \tau_{i}$, then

$$
g_{\max }^{(n)}\left(\underline{\mu^{\prime}}\right)=g_{\max }^{(n)}(\underline{\mu})-1 \text { and } g_{\min }^{(n)}\left(\underline{\mu^{\prime}}\right)=g_{\min }^{(n)}(\underline{\mu})+1 .
$$

(3) If $\nu$ is a word in $\tau_{1}, \cdots, \tau_{b-1}, \mu=\nu \tau_{i}$ and $\mu^{\prime}=\nu \tau_{i} \tau_{i}$, then

$$
g_{\max }^{(n)}\left(\underline{\mu^{\prime}}\right)=g_{\max }^{(n)}(\underline{\mu})+1 \text { and } g_{\min }^{(n)}\left(\underline{\mu^{\prime}}\right)=g_{\min }^{(n)}(\underline{\mu})-1 .
$$

(4) If $\nu$ is a word in $\tau_{1}, \cdots, \tau_{b-1}, \mu_{1}=\nu \tau_{i-1} \tau_{i} \tau_{i-1}, \mu_{2}=\nu \tau_{i}$, and $\mu_{3}=$ $\nu \tau_{i} \tau_{i-1} \tau_{i}$, then

$g_{\max }^{(n)}\left(\underline{\mu_{3}}\right) \leq \max \left\{g_{\max }^{(n)}\left(\underline{\mu_{1}}\right), g_{\max }^{(n)}\left(\underline{\mu_{2}}\right)\right\} \quad$ and $\left.g_{\min }^{(n)} \underline{\left(\mu_{3}\right.}\right) \geq \min \left\{g_{\min }^{(n)}\left(\underline{\mu_{1}}\right), g_{\min }^{(n)}\left(\underline{\mu_{2}}\right)\right\}$.

Proof. (1) We mark the $i$-th strand by a single variable $x_{i}$ of degree 2 , for $i=$ $1, \cdots, b$. A straightforward computation shows that

$$
H_{n}(\underline{\phi})=A_{1} \otimes_{\mathbb{Q}} A_{2} \otimes_{\mathbb{Q}} \cdots \otimes_{\mathbb{Q}} A_{b}
$$

where $A_{i}=\left[\mathbb{Q}\left[x_{i}\right] /\left(x_{i}^{n}\right)\right]\{-n+1\}$. The first part of the lemma follows from this.

(2) Note that the $(i+1)$-th strand in $\mu$ is a circle. We mark it by a single variable $x$ of degree 2 . Denote by $\underline{\hat{\mu}}$ the closed resolved braid obtained from $\underline{\mu}$ by removing the $(i+1)$-th strand. Then

$$
H_{n}(\underline{\mu})=H_{n}(\underline{\hat{\mu}}) \otimes_{\mathbb{Q}} A=\bigoplus_{i=0}^{n-1} H_{n}(\underline{\hat{\mu}})\{-n+1+2 i\},
$$


where $A=\left[\mathbb{Q}[x] /\left(x^{n}\right)\right]\{-n+1\}$. But, from Proposition 29 of 7 , we know that

$$
H_{n}\left(\underline{\mu^{\prime}}\right)=\bigoplus_{i=0}^{n-2} H_{n}(\underline{\hat{\mu}})\{-n+2+2 i\} .
$$

Comparing these two decompositions, and the second part of the lemma follows.

(3) By Proposition 30 of [7, we have

$$
H_{n}\left(\underline{\mu^{\prime}}\right)=H_{n}(\underline{\mu})\{1\} \oplus H_{n}(\underline{\mu})\{-1\},
$$

which implies the third part of the lemma.

(4) By Proposition 32 of [7, we have

$$
H_{n}\left(\underline{\mu_{1}}\right) \oplus H_{n}\left(\underline{\mu_{2}}\right)=H_{n}\left(\underline{\mu_{3}}\right) \oplus H_{n}\left(\underline{\mu_{4}}\right),
$$

where $\mu_{4}=\nu \tau_{i-1}$, and the last part of the lemma follows.

Similar to the proof of Proposition 3.6, we can induct on the weight of a resolved braid using Lemmas 3.5, 3.7 and prove:

Proposition 3.8. If $\mu=\tau_{i_{1}} \cdots \tau_{i_{m}}$ is a word in $\tau_{1}, \cdots, \tau_{b-1}$, then

$$
-(n-1) b-m \leq g_{\min }^{(n)}(\underline{\mu}) \leq g_{\max }^{(n)}(\underline{\mu}) \leq(n-1) b+m .
$$

Proof of inequality (1.2) in Theorem 1.1, Let $B$ be a braid with $b$ strands, $c_{+}$positive crossings, $c_{-}$negative crossings, and writhe $w=c_{+}-c_{-}$. Note that

$$
H_{n}(B)=H\left(H\left(C_{n}(B), d_{m f}\right), d_{\chi}\right)
$$

and

$$
H\left(C_{n}(B), d_{m f}\right)=\bigoplus_{\Gamma} H_{n}(\Gamma)\left\{p_{\Gamma}\right\},
$$

where $\Gamma$ runs through all resolutions of $B$, and $p_{\Gamma} \in \mathbb{Z}$. Let $\Gamma$ be any resolution of $B$. From the construction of $C_{n}(B)$, it's clear that

$$
p_{\Gamma}=(n-1) w+c_{\Gamma,+}-c_{\Gamma,-},
$$

where $c_{\Gamma,+}$ (resp. $c_{\Gamma,-}$ ) is the number of wide edges in $\Gamma$ from positive (resp. negative) crossings in $B$. Let $g$ be the quantum degree of a non-vanishing homogeneous element of $H_{n}(\Gamma)\left\{p_{\Gamma}\right\}$. Then

$$
g_{\min }^{(n)}(\Gamma)+p_{\Gamma} \leq g \leq g_{\min }^{(n)}(\Gamma)+p_{\Gamma} .
$$

But, from Proposition 3.8, we have

$$
-(n-1) b-c_{\Gamma,+}-c_{\Gamma,-} \leq g_{\min }^{(n)}(\Gamma) \leq g_{\max }^{(n)}(\Gamma) \leq(n-1) b+c_{\Gamma,+}+c_{\Gamma,-} .
$$

So

$$
(n-1)(w-b)-2 c_{\Gamma,-} \leq g \leq(n-1)(w+b)+2 c_{\Gamma,+} .
$$

It's clear that $c_{\Gamma,+} \leq c_{+}$and $c_{\Gamma,-} \leq c_{-}$. This shows that, if $g$ is the quantum degree of any non-vanishing homogeneous element of $H\left(C_{n}(B), d_{m f}\right)$, then

$$
(n-1)(w-b)-2 c_{-} \leq g \leq(n-1)(w+b)+2 c_{+} .
$$

Thus,

$$
(n-1)(w-b)-2 c_{-} \leq g_{\min }^{(n)}(B) \leq g_{\max }^{(n)}(B) \leq(n-1)(w+b)+2 c_{+} .
$$




\section{TransVersal KnOt inVariants $\left\{\psi_{n}\right\}$}

In this section, we use the usual notation for braid groups. Denote by $\mathcal{B}_{b}$ the braid group of $b$ strands, and $\sigma_{1}^{ \pm 1}, \cdots, \sigma_{b-1}^{ \pm 1}$ the standard generators of $\mathcal{B}_{b}$, i.e., $\sigma_{i}^{ \pm 1}$ permutes the $i$-th and $(i+1)$-th strands with a $( \pm)$-crossing. Also, denote by $\phi$ the empty word, which is the identity of $\mathcal{B}_{b}$. Two braids represent the same link if and only if one can be changed into the other by the following operations:

- Braid group relations generated by: $\sigma_{i} \sigma_{i}^{-1}=\sigma_{i}^{-1} \sigma_{i}=\phi, \sigma_{i} \sigma_{j}=\sigma_{j} \sigma_{i}$, when $|i-j|>1$, and $\sigma_{i} \sigma_{i+1} \sigma_{i}=\sigma_{i+1} \sigma_{i} \sigma_{i+1}$.

- Conjugations: $\mu \leftrightarrow \eta^{-1} \mu \eta$, where $\mu, \eta \in \mathcal{B}_{b}$.

- Stabilizations and destabilizations:

$$
\begin{cases}\text { positive : } & \mu\left(\in \mathcal{B}_{b}\right) \leftrightarrow \mu \sigma_{b}\left(\in \mathcal{B}_{b+1}\right), \\ \text { negative }: & \mu\left(\in \mathcal{B}_{b}\right) \leftrightarrow \mu \sigma_{b}^{-1}\left(\in \mathcal{B}_{b+1}\right) .\end{cases}
$$

In [2, Bennequin proved that any transversal link is transversally isotopic to a transversal braid. The following theorem from [10, 12] describes when two transversal braids are transversally isotopic.

Theorem 4.1 ([10, 12]). If two transversal braids are transversally isotopic, then one can be changed into the other by a finite sequence of braid group relations, conjugations and positive stabilizations and destabilizations.

Let $\mu$ be a closed braid with $b$ strands, and $\Gamma$ the resolution of $\mu$ so that each crossing of $\mu$ is 0 -resolved as shown in Figure 5. Then $\Gamma$ consists of $b$ concentric circles, and

$$
H_{n}(\Gamma)=A_{1} \otimes_{\mathbb{Q}} A_{2} \otimes_{\mathbb{Q}} \cdots \otimes_{\mathbb{Q}} A_{b}
$$

where $A_{i}=\left[\mathbb{Q}\left[x_{i}\right] /\left(x_{i}^{n}\right)\right]\{-n+1\}$, and $\operatorname{deg} x_{i}=2$ as before. Let $w$ be the writhe of $\mu$. It's clear that the standard isomorphism

$$
f: H_{n}(\Gamma) \stackrel{\cong}{\rightrightarrows} H_{n}(\Gamma)\{(n-1) w\}\left(\subset H\left(C_{n}(\mu), d_{m f}\right)\right)
$$

has quantum degree $(n-1) w$ and maps $H_{n}(\Gamma)$ into cohomological degree 0 . Then the element

$$
\varphi_{n}(\mu)=f\left(x_{1}^{n-1} \otimes \cdots \otimes x_{b}^{n-1}\right)
$$

has quantum degree $(n-1)(w+b)$ and cohomological degree 0 .

Proposition 4.2. $\varphi_{n}(\mu)$ is a cocycle in the chain complex $\left(H\left(C_{n}(\mu), d_{m f}\right), d_{\chi}\right)$ and, up to multiplication by a non-vanishing scalar, its cohomology class $\left[\varphi_{n}(\mu)\right] \in$ $H_{n}(\mu)=H\left(H\left(C_{n}(\mu), d_{m f}\right), d_{\chi}\right)$ is invariant under braid group relations, conjugations and negative stabilizations and destabilizations.

Proof. For a negative crossing $\varsigma$ of $\mu$, denote by $\Gamma_{\varsigma}$ the resolution of $\mu$ obtained by 1-resolving $\varsigma$ and 0-resolving all other crossings. One can easily see that

$$
d_{\chi}\left(H_{n}(\Gamma)\{(n-1) w\}\right) \subset \bigoplus_{\varsigma} H_{n}\left(\Gamma_{\varsigma}\right)\{(n-1) w-1\} \subset H\left(C_{n}(\mu), d_{m f}\right),
$$

where $\varsigma$ runs through all negative crossings of $\mu$. From the second part of Lemma 3.7. we know that the maximal quantum degree of a non-vanishing homogeneous element of $H_{n}\left(\Gamma_{\varsigma}\right)\{(n-1) w-1\}$ is $(n-1)(w+b)-2$. But $d_{\chi}$ preserves the quantum grading. So $d_{\chi}\left(\varphi_{n}(\mu)\right)$ is a homogeneous element of degree $(n-1)(w+b)$. This implies that $d_{\chi}\left(\varphi_{n}(\mu)\right)=0$, and, hence, $\varphi_{n}(\mu)$ is a cocycle.

Next, we prove the invariance of $\left[\varphi_{n}(\mu)\right]$ under the local moves depicted in Figure 11. which implies the second part of the proposition. 


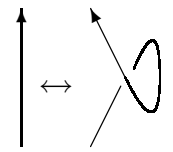

$\mu_{0} \quad \mu_{1}$

(i)

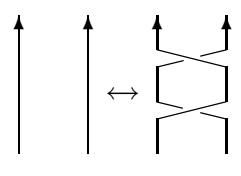

$\mu_{0}$

$\left(\mathrm{ii}_{a}\right)$

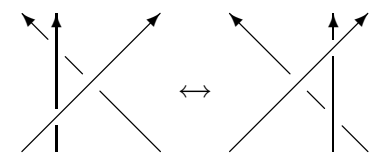

$\mu_{0}$ $\mu_{1}$

(iii)

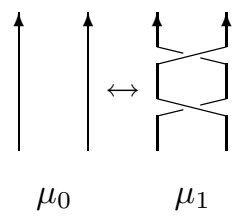

$\left(\mathrm{ii}_{b}\right)$

FiguRe 11. Local moves

Remark 4.3. All together, there are 6 braidlike Reidemeister type 3 local moves to deal with. These correspond to the 6 possible orderings of the three branches involved. But each of the other 5 braidlike Reidemeister type 3 local moves is related to move (iii) by braidlike Reidemeister type 2 local moves. So we only need to prove the invariance under move (iii) here.

Invariance under move (i). Consider the planar diagrams in Figure 12 ,

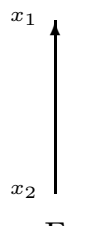

$\Gamma$

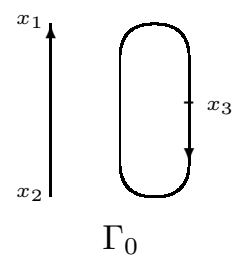

$\Gamma_{0}$

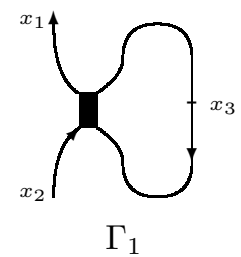

$\Gamma_{1}$

FIGURE 12. Local diagrams related to move (i)

The local complex associated to $\mu_{0}$ is

$$
0 \rightarrow H_{n}(\Gamma) \rightarrow 0,
$$

and the local complex associated to $\mu_{1}$ is

$$
0 \rightarrow H_{n}\left(\Gamma_{0}\right)\{1-n\} \rightarrow H_{n}\left(\Gamma_{1}\right)\{-n\} \rightarrow 0 .
$$

Note that $H_{n}\left(\Gamma_{0}\right)=H_{n}(\Gamma) \otimes A_{3}$, where $A_{3}=\left[\mathbb{Q}\left[x_{3}\right] /\left(x_{3}^{n}\right)\right]\{-n+1\}$ and $\operatorname{deg} x_{3}=2$.

There is a homomorphism $A_{3} \stackrel{\varepsilon}{\rightarrow} \mathbb{Q}$ of degree $1-n$ given by

$$
\varepsilon\left(x_{3}^{j}\right)= \begin{cases}1, & \text { if } j=n-1 ; \\ 0, & \text { otherwise. }\end{cases}
$$

This induces a quantum degree preserving homomorphism $\varepsilon: H_{n}\left(\Gamma_{0}\right)\{1-n\} \rightarrow$ $H_{n}(\Gamma)$, which, in turn, induces a bi-degree preserving homomorphism of complexes

$$
\begin{aligned}
& 0 \rightarrow H_{n}\left(\Gamma_{0}\right)\{1-n\} \rightarrow H_{n}\left(\Gamma_{1}\right)\{-n\} \rightarrow 0 \\
& 0 \rightarrow H_{n}(\Gamma) \quad \rightarrow \quad 0 \text {. }
\end{aligned}
$$


It's clear that this chain map maps $\varphi_{n}\left(\mu_{1}\right)$ to $\varphi_{n}\left(\mu_{0}\right)$, and, from Section 8 of [7, we know that this chain map induces (a non-zero multiple of) the standard isomorphism from $H_{n}\left(\mu_{1}\right)$ to $H_{n}\left(\mu_{0}\right)$. Thus, $\left[\varphi_{n}(\mu)\right]$ is invariant under local move (i) up to multiplication by a non-vanishing scalar.

Invariance under moves $\left(\mathbf{i i}_{a}\right)$ and $\left(\mathbf{i i}_{b}\right)$. The proofs for these two moves are almost identical. So we only prove the invariance under move $\left(\mathrm{ii}_{a}\right)$.

Consider the planar diagrams in Figure 13

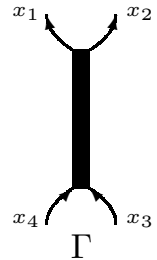

$\Gamma_{00}$
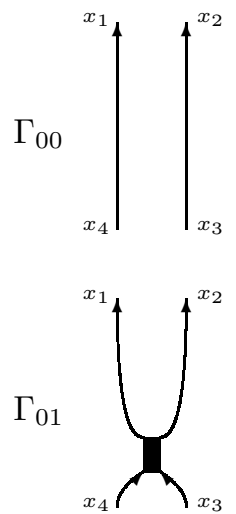
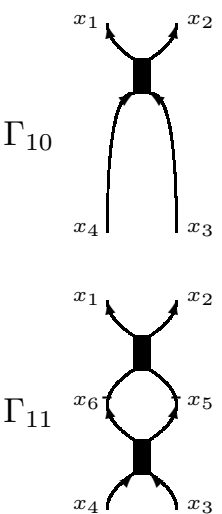

Figure 13. Local diagrams related to move $\left(\mathrm{ii}_{a}\right)$

The local complex associated to $\mu_{0}$ is

$$
0 \rightarrow H_{n}\left(\Gamma_{00}\right) \rightarrow 0,
$$

and the local complex associated to $\mu_{1}$ is

$$
0 \rightarrow H_{n}\left(\Gamma_{01}\right)\{1\} \rightarrow H_{n}\left(\Gamma_{00}\right) \oplus H_{n}\left(\Gamma_{11}\right) \rightarrow H_{n}\left(\Gamma_{10}\right)\{-1\} \rightarrow 0 .
$$

Let

$$
\pi_{11}: H_{n}\left(\Gamma_{00}\right) \oplus H_{n}\left(\Gamma_{11}\right) \rightarrow H_{n}\left(\Gamma_{11}\right)
$$

be the natural projection. Consider the quantum degree preserving homomorphisms

$$
f_{1}=\pi_{11} \circ d_{\chi}: H_{n}\left(\Gamma_{01}\right)\{1\} \rightarrow H_{n}\left(\Gamma_{11}\right)
$$

and

Note that

$$
f_{2}=\left.d_{\chi}\right|_{H_{n}\left(\Gamma_{11}\right)}: H_{n}\left(\Gamma_{11}\right) \rightarrow H_{n}\left(\Gamma_{10}\right)\{-1\} .
$$

$$
H_{n}\left(\Gamma_{11}\right) \cong H_{n}(\Gamma)\{1\} \oplus H_{n}(\Gamma)\{-1\}
$$

Let

$$
f_{10}: H_{n}\left(\Gamma_{01}\right)\{1\} \rightarrow H_{n}(\Gamma)\{1\} \text { and } f_{21}: H_{n}(\Gamma)\{-1\} \rightarrow H_{n}\left(\Gamma_{10}\right)\{-1\}
$$

be the obvious maps induced by $f_{1}, f_{2}$ and the above isomorphism. Note that $\Gamma_{01}$ and $\Gamma_{10}$ are isotopic to $\Gamma$. In Section 8 of [7, M. Khovanov and L. Rozansky showed that $f_{10}$ and $f_{21}$ are non-zero multiples of the natural isomorphisms

$$
H_{n}\left(\Gamma_{01}\right)\{1\} \stackrel{\cong}{\rightrightarrows} H_{n}(\Gamma)\{1\} \text { and } H_{n}(\Gamma)\{-1\} \stackrel{\cong}{\rightrightarrows} H_{n}\left(\Gamma_{10}\right)\{-1\} \text {. }
$$


Define the submodule $A \subset H_{n}\left(\Gamma_{00}\right) \oplus H_{n}(\Gamma)\{-1\} \subset H_{n}\left(\Gamma_{00}\right) \oplus H_{n}\left(\Gamma_{11}\right)$ by

$$
A=\left\{\left(a,-f_{21}^{-1} \circ d_{\chi}(a)\right) \mid a \in H_{n}\left(\Gamma_{00}\right)\right\}
$$

and the submodule $B \subset H_{n}\left(\Gamma_{00}\right) \oplus H_{n}\left(\Gamma_{11}\right)$ by

$$
B=d_{\chi}\left(H_{n}\left(\Gamma_{01}\right)\{1\}\right) .
$$

Then

$$
H_{n}\left(\Gamma_{00}\right) \oplus H_{n}\left(\Gamma_{11}\right)=A \oplus B \oplus H_{n}(\Gamma)\{-1\} .
$$

It's easy to see that $f_{21}=\left.d_{\chi}\right|_{H_{n}(\Gamma)\{-1\}}$. Thus, the local complex associated to $\mu_{1}$ splits into the direct sum of the following chain complexes:

$$
\begin{aligned}
& \begin{array}{ccccc}
0 & \rightarrow & A & \rightarrow & 0,
\end{array} \\
& \left.0 \rightarrow H_{n}\left(\Gamma_{01}\right)\{1\}\right\} \stackrel{\rightrightarrows}{\longrightarrow} \quad B \quad \rightarrow \quad 0, \\
& 0 \quad \rightarrow H_{n}(\Gamma)\{-1\} \stackrel{\cong}{\rightrightarrows} H_{n}\left(\Gamma_{10}\right)\{-1\} \rightarrow 0 .
\end{aligned}
$$

The second and third chain complexes give rise to trivial cohomologies, and the standard isomorphism from $H_{n}\left(\mu_{0}\right)$ to $H_{n}\left(\mu_{1}\right)$ is induced by (a non-zero scalar multiple of) the isomorphism of chain complexes

$$
F:\left[0 \rightarrow H_{n}\left(\Gamma_{00}\right) \rightarrow 0\right] \stackrel{\cong}{\rightarrow}[0 \rightarrow A \rightarrow 0]
$$

given by

$$
F(a)=\left(a,-f_{21}^{-1} \circ d_{\chi}(a)\right) .
$$

Since $d_{\chi}\left(\varphi_{n}\left(\mu_{0}\right)\right)=0$, it's easy to see that $F\left(\varphi_{n}\left(\mu_{0}\right)\right)=\varphi_{n}\left(\mu_{1}\right)$. Thus, $\left[\varphi_{n}(\mu)\right]$ is invariant under local move $\left(\mathrm{ii}_{a}\right)$ up to multiplication by a non-vanishing scalar.

Invariance under moves (iii). The proof in [7] of the invariance of $H_{n}$ under move (iii) is much more complex than that under moves (i) and (ii). But, if we first use D. Bar-Natan's algebraic trick in [1] to reformulate the description of the isomorphism of cohomologies, then it is quite easy to establish the invariance of $\left[\varphi_{n}(\mu)\right]$ under this move.
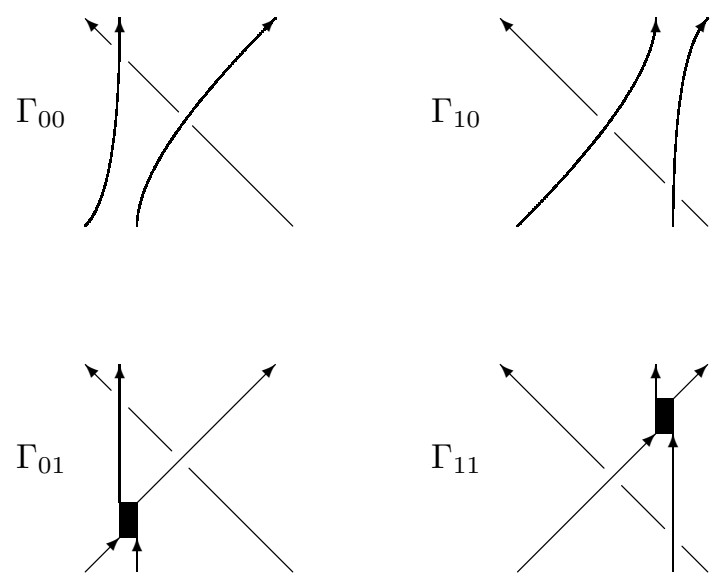

Figure 14. Local diagrams related to move (iii)

Consider the partially resolved planar diagrams in Figure 14. We extend the definition of the (double) chain complex $\left(C_{n}, d_{m f}, d_{\chi}\right)$ to these diagrams. Then, for 
$i=0,1$, the chain complex $\left(H\left(C_{n}\left(\mu_{i}\right), d_{m f}\right), d_{\chi}\right)$ is the mapping cone of the chain map

$$
\chi_{i 1}:\left(H\left(C_{n}\left(\Gamma_{i 1}\right), d_{m f}\right), d_{\chi}\right) \rightarrow\left(H\left(C_{n}\left(\Gamma_{i 0}\right), d_{m f}\right), d_{\chi}\right),
$$

where $\chi_{i 1}$ is induced by the $\chi_{1}^{(n)}$-map associated to the wide edge in $\Gamma_{i 1}$. Note that $\Gamma_{00}$ and $\Gamma_{10}$ are the same diagram, which we denote by $\Gamma$. Although formulated differently in [7, M. Khovanov and L. Rozansky actually constructed a chain complex $\mathfrak{C}$ which is a strong deformation retract of both $\left(H\left(C_{n}\left(\Gamma_{01}\right), d_{m f}\right), d_{\chi}\right)$ and $\left(H\left(C_{n}\left(\Gamma_{11}\right), d_{m f}\right), d_{\chi}\right)$ with corresponding inclusion maps

$$
F_{i}: \mathfrak{C} \rightarrow\left(H\left(C_{n}\left(\Gamma_{i 1}\right), d_{m f}\right), d_{\chi}\right), i=0,1,
$$

so that $\chi_{01} \circ F_{0}=\chi_{11} \circ F_{1}$ as homomorphisms from $\mathfrak{C}$ to $\left(H\left(C_{n}(\Gamma), d_{m f}\right), d_{\chi}\right)$. Thus, the following squares commute:

$$
\left.\begin{array}{ccccc}
\left(H\left(C_{n}\left(\Gamma_{00}\right), d_{m f}\right), d_{\chi}\right) & \stackrel{\mathrm{id}}{\longrightarrow} & \left(H\left(C_{n}(\Gamma), d_{m f}\right), d_{\chi}\right) & \stackrel{\mathrm{id}}{\longleftarrow} & \left(H\left(C_{n}\left(\Gamma_{10}\right), d_{m f}\right), d_{\chi}\right) \\
\chi_{01} \uparrow & \chi \uparrow & \chi_{11} \uparrow
\end{array}\right),
$$

where id is the natural identity homomorphism, and $\chi=\chi_{01} \circ F_{0}=\chi_{11} \circ F_{1}$.

By Lemma 4.5 of [1, for $i=0,1$, the maps id and $F_{i}$ induce an isomorphism $\mathcal{F}_{i}$ from $H_{n}\left(\mu_{i}\right)$ to the cohomology of the mapping cones of $\chi: \mathfrak{C} \rightarrow$ $\left(H\left(C_{n}(\Gamma), d_{m f}\right), d_{\chi}\right)$. Then the isomorphism

$$
\mathcal{F}=\mathcal{F}_{1}^{-1} \circ \mathcal{F}_{0}: H_{n}\left(\mu_{0}\right) \rightarrow H_{n}\left(\mu_{1}\right)
$$

is (a multiple of) the standard isomorphism. It's clear that

$$
\begin{aligned}
& \varphi\left(\mu_{0}\right) \in H\left(C_{n}\left(\Gamma_{00}\right), d_{m f}\right) \subset H\left(C_{n}\left(\mu_{0}\right), d_{m f}\right), \\
& \varphi\left(\mu_{1}\right) \in H\left(C_{n}\left(\Gamma_{10}\right), d_{m f}\right) \subset H\left(C_{n}\left(\mu_{1}\right), d_{m f}\right),
\end{aligned}
$$

and

$$
\operatorname{id}\left(\varphi\left(\mu_{0}\right)\right)=\varphi\left(\mu_{1}\right)
$$

where id $: H\left(C_{n}\left(\Gamma_{00}\right), d_{m f}\right) \rightarrow H\left(C_{n}\left(\Gamma_{10}\right), d_{m f}\right)$ is the natural identity homomorphism. This implies that

$$
\mathcal{F}\left(\left[\varphi\left(\mu_{0}\right)\right]\right)=\left[\varphi\left(\mu_{1}\right)\right]
$$

which shows that $\left[\varphi_{n}(\mu)\right]$ is invariant under local move (iii) up to multiplication by a non-vanishing scalar.

Let $L$ be a transversal link in the standard contact $S^{3}$. We transversally isotope it into a transversal braid.

Definition 4.4. $\psi_{n}(L)=\left[\varphi_{n}(\bar{L})\right] \in H_{n}(\bar{L})$.

Proof of Theorem 1.5. From Theorem 4.1 and Proposition 4.2. we know that, up to multiplication by a non-zero scalar, $\psi_{n}(L)$ is independent of the transversal braid representation and is therefore invariant under transversal isotopy. From the construction of $\psi_{n}(L)=\left[\varphi_{n}(\bar{L})\right]$, it's clear that it has cohomological degree 0 and quantum degree $(n-1)(-w+b)=-(n-1) s l(L)$, where $w$ is the writhe of $L$, and $b$ is the number of strands of $L$. Compare our construction with that by Plamenevskaya in [11. One easily sees that $\psi_{2}(L)$ is identified with $\psi(L)$ under the isomorphism $H_{2}(\bar{L}) \cong \mathcal{H}(L) \otimes_{\mathbb{Z}} \mathbb{Q}$.

Next we generalize Plamenevskaya's result for quasi-positive transversal braids, which completes the proof of Corollary 1.6 . 
Proposition 4.5 (Compare Theorem 4 of [11]). Let $\mu \in \mathcal{B}_{b}$ be a closed braid, and $\mu^{\prime}=\mu \sigma_{i}^{-1}$, where $1 \leq i \leq b-1$. Then there is a homomorphism $f: H_{n}\left(\mu^{\prime}\right) \rightarrow$ $H_{n}(\mu)$ of quantum degree $n-1$ such that $f\left(\left[\varphi_{n}\left(\mu^{\prime}\right)\right]\right)=\left[\varphi_{n}(\mu)\right]$. In particular, this implies that the $\psi_{n}$ invariant of a quasi-positive transversal braid is non-vanishing.

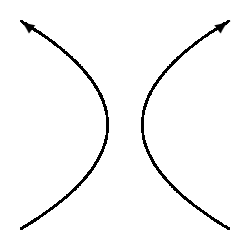

$\Gamma_{0}$

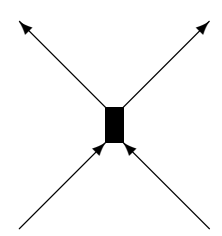

$\Gamma_{1}$

FiguRE 15. Diagrams $\Gamma_{0}$ and $\Gamma_{1}$

Proof. The local chain complex associated to $\sigma_{i}^{-1}$ in $\left(H_{n}\left(\mu^{\prime}, d_{m f}\right), d_{\chi}\right)$ is

$$
0 \rightarrow H_{n}\left(\Gamma_{0}\right)\{1-n\} \rightarrow H_{n}\left(\Gamma_{1}\right)\{-n\} \rightarrow 0,
$$

and the corresponding local complex in $\left(H_{n}\left(\mu, d_{m f}\right), d_{\chi}\right)$ is

$$
0 \rightarrow H_{n}\left(\Gamma_{0}\right) \rightarrow 0 .
$$

The identity map of $H_{n}\left(\Gamma_{0}\right)$ induces a local chain map of quantum degree $n-1$ :

$$
\begin{aligned}
& 0 \rightarrow H_{n}\left(\Gamma_{0}\right)\{1-n\} \rightarrow H_{n}\left(\Gamma_{1}\right)\{-n\} \rightarrow 0 \\
& 0 \rightarrow H_{n}\left(\Gamma_{0}\right) \quad \rightarrow \quad 0 .
\end{aligned}
$$

Let $f: H_{n}\left(\mu^{\prime}\right) \rightarrow H_{n}(\mu)$ be the homomorphism induced by the above local chain map. It's clear that $f\left(\left[\varphi_{n}\left(\mu^{\prime}\right)\right]\right)=\left[\varphi_{n}(\mu)\right]$.

Now let $L$ be a quasi-positive transversal braid of $b$ strands, i.e., $L$ is represented by a braid word of the form

$$
\nu=\mu_{1} \sigma_{i_{1}} \mu_{1}^{-1} \cdots \mu_{k} \sigma_{i_{k}} \mu_{k}^{-1} .
$$

Then $\bar{L}$ is represented by the word

$$
\bar{\nu}=\bar{\mu}_{1} \sigma_{i_{1}}^{-1} \bar{\mu}_{1}^{-1} \cdots \bar{\mu}_{k} \sigma_{i_{k}}^{-1} \bar{\mu}_{k}^{-1},
$$

where $\bar{\mu}_{j}$ is the mirror image of $\mu_{j}$. From the first half of the proposition and the invariance of $\left[\varphi_{n}\right]$, we know that there is a homomorphism $F: H_{n}(\bar{L}) \rightarrow H_{n}(\phi)$ such that $F\left(\left[\varphi_{n}(\bar{\nu})\right]\right)=\left[\varphi_{n}(\phi)\right] \neq 0$, where $\phi$ is the empty word in $b$ strands. Thus, $\psi_{n}(L)=\left[\varphi_{n}(\bar{\nu})\right] \neq 0$.

Some other properties of Plamenevskaya's $\psi$ invariant generalize to $\psi_{n}$ too. For example:

Proposition 4.6 (Compare Proposition 3 of [11]). Let $\mu \in \mathcal{B}_{b}$ be a braid with $b$ strands. If there is an $i \in\{1, \cdots, b-1\}$ such that $\sigma_{i}$ occurs in the word $\mu$, but $\sigma_{i}^{-1}$ does not, then $\left[\varphi_{n}(\mu)\right]=0, \forall n \geq 2$. In particular, if a transversal link $L$ is a transversal stabilization of another transversal link, then $\psi_{n}(L)=0, \forall n \geq 2$. 
Proof. Fix a crossing $\sigma_{i}$ in $\mu$. Let $\Gamma_{-1}$ be the resolution of $\mu$ obtained by 1-resolving $\sigma_{i}$ and 0-resolving all other crossings, and let $\Gamma_{0}$ be the resolution of $\mu$ obtained by 0 -resolving all the crossings. For a negative crossing $\varsigma$ of $\mu$, let $\Gamma_{\varsigma}$ be the resolution of $\mu$ obtained by 1-resolving $\sigma_{i}$, $\varsigma$ and 0-resolving all other crossings. Since $\mu$ does not contain $\sigma_{i}^{-1}, \Gamma_{\varsigma}$ is a resolved closed braid of the form $\tau_{i} \tau_{j}$, where $j \neq i$. Then, in the chain complex $\left(H\left(C_{n}(\mu), d_{m f}\right), d_{\chi}\right)$, we have

$$
d_{\chi}\left(H_{n}\left(\Gamma_{-1}\right)\{(n-1) w+1\}\right) \subset H_{n}\left(\Gamma_{0}\right)\{(n-1) w\} \oplus\left(\bigoplus_{\varsigma} H_{n}\left(\Gamma_{\varsigma}\right)\{(n-1) w\}\right),
$$

where $w$ is the writhe of $\mu$, and $\varsigma$ runs through all negative crossings of $\mu$. Let

$$
\pi_{0}: H_{n}\left(\Gamma_{0}\right)\{(n-1) w\} \oplus\left(\bigoplus_{\varsigma} H_{n}\left(\Gamma_{\varsigma}\right)\{(n-1) w\}\right) \rightarrow H_{n}\left(\Gamma_{0}\right)\{(n-1) w\}
$$

be the natural projection. Then it's clear that

$$
\pi_{0} \circ d_{\chi}=\chi_{1}: H_{n}\left(\Gamma_{-1}\right)\{(n-1) w+1\} \rightarrow H_{n}\left(\Gamma_{0}\right)\{(n-1) w\},
$$

where $\chi_{1}$ is induced by the $\chi_{1}^{(n)}$-map related to the crossing $\sigma_{i}$. From Proposition 29 of [7, we know that there is a non-zero homogeneous element $\eta \in$ $H_{n}\left(\Gamma_{-1}\right)\{(n-1) w+1\}$ of quantum degree $(n-1)(w+b)$ such that $\chi_{1}(\eta)=$ $\varphi_{n}(\mu) \in H_{n}\left(\Gamma_{0}\right)\{(n-1) w\}$. By (1) and (2) of Lemma 3.7. we have that $g_{\max }^{(n)}\left(\Gamma_{\varsigma}\right)=$ $(n-1) b-2$ for any negative crossing $\varsigma$ of $\mu$. So the maximal quantum degree of a non-zero homogeneous element of $H_{n}\left(\Gamma_{\varsigma}\right)\{(n-1) w\}$ is $(n-1)(w+b)-2$. Since $d_{\chi}$ preserves the quantum degree, the projection of $d_{\chi}(\eta)$ onto $H_{n}\left(\Gamma_{\varsigma}\right)\{(n-1) w\}$ is zero for any negative crossing $\varsigma$ of $\mu$. This implies that $d_{\chi}(\eta)=\varphi_{n}(\mu)$. Thus, $\varphi_{n}(\mu)$ is a coboundary, and $\left[\varphi_{n}(\mu)\right]=0$.

If $L$ is a transversal stabilization of another transversal link, then there is a $b \geq 2$, such that $L$ is transversally isotopic to a transversal braid given by a braid word $\mu \in \mathcal{B}_{b}$, where $\mu$ contains one $\sigma_{b-1}^{-1}$ and no $\sigma_{b-1}$. Therefore, $\psi_{n}(L)=\left[\varphi_{n}(\bar{\mu})\right]=$ 0 .

\section{ACKNOWLEDGMENTS}

The author's interest in finding new bounds for the self-linking number was inspired by Matt Hedden's work on Legendrian knots and the knot Floer homology. The basic idea of Theorem 1.1 was conceived during the Holomorphic Curves Workshop held at IAS in May and June of 2005. The author would like to thank the organizers for their hospitality, and Matt Hedden for interesting conversations. The author would also like to thank the referee for many helpful comments on an earlier draft of this paper.

\section{REFERENCES}

1. D. Bar-Natan, Khovanov's homology for tangles and cobordisms, Algebr. Geom. Topol. 9 (2005), 1443-1499 (electronic). MR2174270 (2006g:57017)

2. D. Bennequin, Entrelacements et équations de Pfaff, Astérisque 107-108 (1983), 87-161. MR.753131 (86e:58070)

3. E. Ferrand, On Legendrian knots and polynomial invariants, Proc. Amer. Math. Soc. 130 (2002), no. 4, 1169-1176 (electronic). MR1873793 (2002j:57047)

4. J. Franks, R. F. Williams, Braids and the Jones polynomial, Trans. Amer. Math. Soc. 303 (1987), no. 1, 97-108. MR896009 (88k:57006)

5. D. Fuchs, S. Tabachnikov, Invariants of Legendrian and transverse knots in the standard contact space, Topology 36 (1997), no. 5, 1025-1053. MR1445553(99a:57006) 
6. M. Khovanov, A categorification of the Jones polynomial, Duke Math. J. 101 (2000), no. 3, 359-426. MR.1740682 (2002j:57025)

7. M. Khovanov, L. Rozansky, Matrix factorizations and link homology, arXiv:math.QA/ 0401268 .

8. M. Khovanov, L. Rozansky, Matrix factorizations and link homology II, arXiv:math.QA/ 0505056 .

9. H. Morton, Seifert circles and knot polynomials, Math. Proc. Cambridge Philos. Soc. 99 (1986), no. 1, 107-109. MR809504 (87c:57006)

10. S. Orevkov, V. Shevchishin, Markov theorem for transversal links, J. Knot Theory Ramifications 12 (2003), no. 7, 905-913. MR.2017961 (2004j:57011)

11. O. Plamenevskaya, Transverse knots and Khovanov homology, Math. Res. Lett. 13 (2006), 571-586. MR2250492 (2007d:57043)

12. N. Wrinkle, The Markov Theorem for transverse knots, arXiv:math.GT/0202055.

Department of Mathematics and Statistics, Lederle Graduate Research Tower, 710 North Pleasant Street, University of Massachusetts, Amherst, Massachusetts 010039305

E-mail address: wu@math.umass.edu

Current address: Department of Mathematics, The George Washington University, Monroe Hall, Room 240, 2115 G Street, N.W., Washington, DC 20052

E-mail address: haowu@gwu.edu 